\title{
Die Bedeutung indigenen Wissens für die Geschichte des Kautschuks
}

\author{
Von Jens Soentgen
}

\begin{abstract}
Überblick
Die Bedeutung wissenschaftlichen Wissens in der Geschichte des Kautschuks ist in vielen Untersuchungen etwa zur Erfindung der Vulkanisation und des Synthesekautschuks differenziert aufgearbeitet; zur Bedeutung indigenen Wissens für die Geschichte dieses Materials ist seit Erland Nordenskiölds Studien kaum mehr geforscht worden. Im Folgenden weise ich nach, dass die indigenen Völker Süd- und Mittelamerikas nicht nur über botanisches Wissen hinsichtlich latexführender Pflanzen verfügten, sondern auch über ein entwickeltes chemisch-technologisches Prozesswissen. Kernstück dieses chemischtechnologischen Wissens ist eine indigene Methode, durch eine bestimmte Form des Räucherns den Kautschuk zu stabilisieren. Dieses Verfahren kann als funktionales Äquivalent zur Vulkanisation bezeichnet werden. So konnten robuste Kautschukprodukte hergestellt werden, die gegen Fäulnis und gegen Sonnenlicht und Hitze weitaus unempfindlicher waren als bloß getrockneter Latex. Durch die Erfindung und industrielle Umsetzung der Vulkanisation Mitte des 19. Jahrhunderts wurde das indigene Wissen zur Fertigung stabiler Gummiprodukte teilweise entbehrlich. Entsprechend waren etwa ab dieser Zeit die Indianer nicht mehr als Produzenten haltbarer Gummiprodukte tätig, sondern nur mehr als Beschaffer des Rohstoffs. Ursprünglich indigenes Wissen wird jedoch auch heute noch weltweit genutzt, da die Räucherung als Vorbehandlung des Kautschuks praktiziert wird. Zugleich gibt es in Nordwestamazonien Versuche, das indigene Kautschukhandwerk zu revitalisieren.
\end{abstract}

\begin{abstract}
The relevance of scientific knowledge to the history of rubber has been demonstrated in many studies, e.g., on vulcanization or synthetic rubber. The relevance of indigenous knowledge, however, has not been analysed with similar rigor. In the following I will show, that Amerindian peoples not only had botanical knowledge concerning rubber plants, but also technological knowledge of the chemical process. They possessed a method for stabilizing rubber by curing. This method may be defined as a functional equivalent to vulcanization. Thus, they were able to produce rubber goods, that were resistant to microbial attacks, sunlight and heat. The invention and industrial use of vulcanization in the 19th century made indigenous knowledge partly dispensable. Correspondingly, Amerindians from that time on no longer practiced as rubber craftsmen in making rubber products,
\end{abstract}


but only as rubber harvesters. Indigenous knowledge is also in use nowadays, as even in industrial supply chains curing remains an important stage in the processing of the material. At the same time, in Northwest-Amazonia, we encounter efforts to revitalize the original rubber craft.

\section{Staunen über Kautschuk}

Gummi - oder Kautschuk - zählt zweifellos zu den außergewöhnlichsten neuen Materialien, das in Europa seit der Entdeckung der Metalle bekannt wurde. Kautschuk ist ein Feststoff und lässt sich doch wie die Luft in alle Richtungen dehnen, um dann wieder seine ursprüngliche Gestalt anzunehmen. Er wurde zuerst von indigenen Völkern Süd- und Mittelamerikas genutzt und war wie der Jesuit Bernabé Cobo (1582-1657), der 61 Jahre in Mittel- und Südamerika verbrachte, ,bien conocido en todas las Indias“, ${ }^{1}$ wohlbekannt in ganz Amerika. Gonzalo Fernández de Oviedo, der in seiner Jugend Christoph Columbus noch persönlich kennengelernt hatte, erzählt von den Bällen der Indianer: „Estas pelotas saltan [...] sin comparación, porque de solo soltalla de la mano en tierra, suben mucho más para arriba, e dan un salto, e otro e otro, y muchos ${ }^{\text {‘2 }}$ (Diese Bälle hüpfen unvergleichlich, denn wenn sie auf den Boden geworfen werden, hüpfen sie höher wieder empor und tun einen weiteren Hüpfer und noch einen und noch einen und viele). Der Kautschuk war in den Augen der Entdecker und der Conquistadoren auf dem Gebiet der Stoffe das, was die neuweltliche, sensible Mimose auf dem Gebiet der Botanik war, ein wunderbares Paradox. Bartolomé de las Casas, der die Bälle und auch die Ballspiele der Indianer ebenfalls eingehend beschreibt, behauptet gar, die Bälle hüpften nahezu eine Viertelstunde lang: „,casi un cuarto de hora de saltar no cesa“. ${ }^{3}$ Schon Christoph Columbus hatte, wie Las Casas berichtet, aus der Neuen Welt einen solchen Ball „tan grande como una botija“, groß wie ein Krug, nach Sevilla mitgebracht. ${ }^{4}$ Dieser Kautschukball, der der bisherigen Kautschukhistoriografie entgangen ist, dürfte das allererste Kautschukprodukt gewesen sein, welches den Boden der Alten Welt erreichte.

Die völlig neuartigen Eigenschaften faszinierten auch die Naturforscher des 19. Jahrhunderts:

„[Das Federharz ist] eine durchaus eigenthümliche Substanz, die, weder den Harzen noch den Gummiarten angehörend, ganz isolirt dasteht. Seine außerordentliche [...] Elastizität reihet es gewissermaßen den lebenden Organismen an“. 5

1 Bernabé Cobo, Historia del Nuevo Mundo. Primera Parte, 1653, Nova edicion: Biblioteca de Autores Espanoles, Tomo 91, Madrid 1964, Kap. XXIX, S. 268.

2 Gonzalo Fernández de Oviedo y Valdés, Historia General Y Natural de las Indias. Primera Parte, La Real Academia de la Historia, Madrid 1992 (1535), Bd. VI, Kap. II, S. 145.

3 Fr. Bartolomé de Las Casas, Los Indios de México y Nueva Espana. Antología. Ed. Edmundo o'Gorman, Jorge Alberto Manrique, Mexico, Editorial Porrua, 1971, S. 23.

4 Ebd.

5 Friedrich Lüdersdorff, Das Auflösen und Wiederherstellen des Federharzes, genannt: Gummi elasticum; zur Darstellung luft- und wasserdichter Gegenstände, Berlin 1832, S. 15. 
Charles Goodyear spürte im Kautschuk sogar die Weisheit Gottes:

„There is probably no other inert substance, the properties of which excite in the human mind, when first called to examine it, an equal amount of curiosity, surprise, and admiration. Who can examine, and reflect upon this property of gum-elastic, without adoring the wisdom of the Creator?" ${ }^{2}$

Die Mischung aus gutmütiger Weichheit und rüstiger Elastizität verleiht dem Stoff eine komische Gestik, er kann zappeln und wackeln wie ein Clown, ein Eindruck, den Gottfried Semper umschrieb, indem er den Kautschuk als „Affe unter den Nutzmaterien" bezeichnete. ${ }^{7}$ Der Eindruck der Lebendigkeit wird durch die hautartige Haptik des Kautschuks verstärkt.

Ohne Kautschuk würden wir heute in einer anderen Welt leben, in der nicht nur das Auto und das Fahrrad fehlen dürften, für deren Reifen heute der Hauptteil des produzierten Gummis verwandt wird. Der Gummi-Industrielle Paul W. Litchfield, damals Präsident der Goodyear Tire \& Rubber, fasste dies 1939 in folgendem Vergleich zusammen:

„Think of our industrial structure as a living thing, the skeleton of which is composed of metal and cement, the arterial system of which carries a life stream of oil, and the flexing muscles and sinews of which are of rubber." ${ }^{\text {(8 }}$

\section{Indigenes Wissen}

In vielen Detailuntersuchungen ist die Rolle von Wissen in der Geschichte des Kautschuks bzw. Gummis aufgearbeitet worden und auch vergleichende Untersuchungen über den Einfluss verschiedener Wissensregime und Wissenstransfers wurden durchgeführt. ${ }^{9}$ Dabei geht es jedoch stets um die Wissensproduktion europäischer oder nordamerikanischer Erfinder, Techniker oder Chemiker. Der Begriff des indigenen Wissens ist dagegen in der Technik- und Wissenschaftsgeschichte des Kautschuks bislang kaum eingeführt. In der ansonsten wichtigen und aufgrund der darin abgedruckten Quellen zur frühen

6 Charles Goodyear, Gum-Elastic and its Varieties, with a Detailed Account of its Applications and Uses, and of the Discovery of Vulcanization, Bd. I, New Haven 1855. Zitiert nach: A Centennial volume of the writings of Charles Goodyear and Thomas Hancock, Boston 1939, S. 23.

7 Gottfried Semper, Der Stil in den technischen und tektonischen Künsten oder Praktische Aesthetik, Bd. 1: Textile Kunst, München 1878, S. 105-112, hier S. 105.

8 Paul Litchfield talk to the American Chemical Society, Boston, Massachusetts, September 13, 1939, zitiert nach: John Tully, The Devil's Milk. A Social History of Rubber, New York 2012, S. 17.

9 Siehe Jochen Streb, Der transatlantische Wissenstransfer auf dem Gebiet der Synthesekautschukforschung in Krieg und Frieden. Freiwillige Kooperationen und erzwungene Reparationen (1926-1954), in: Technikgeschichte 71, 2004, S. 283-303. Vgl. auch die Spezialbibliografie von Joan C. Long, The History of Rubber - A Survey of Sources about the History of Rubber, in: Rubber Chemistry and Technology 74, 2001, S. 493-508. 
nordamerikanischen Industriegeschichte des Kautschuks auch heute noch unentbehrlichen Dissertation von Nancy Norton liest man gleich zu Beginn:

„Rubber, like many things American, ,begins“ with Columbus. But to the popular mind it starts three hundred and fifty years later with Charles Goodyear and his ,metallic gum elastic' vulcanized rubber. [...] Many others had worked with the sticky unreliable vegetable resin from South America [...] but only Charles Goodyear grasped the significance of the combination [of rubber and sulphur, J.S.]." ${ }^{\circ 10}$

Dieses Zitat ist zwar extrem, in der Tendenz aber keinesfalls untypisch für die Kautschukliteratur. Indigene Erkenntnisse werden in einer Perspektive, die sich auf europäische oder nordamerikanische Gestalten und ihre Leistungen konzentriert, nicht selten ignoriert, allenfalls als Vorstufe gewürdigt und in diesem Fall meist naturalisiert als „traditionelle“ Techniken. Sie kommen in den Blick als Teil der Sitten und Gebräuche, und werden nicht als technische oder kognitive Leistungen dargestellt. Nicht wenige Kautschukhistorien thematisieren intensiv die bisweilen blutigen mesoamerikanischen Ballspiele, sprechen aber nicht von den Erfindungen, die nötig waren, um jene Bälle fertigen zu können. Untersuchungen zu kognitiven Leistungen indigener Völker in Bezug auf die Nutzung und Verarbeitung von Latex und Kautschuk sind selten. ${ }^{11}$

Der Begriff des indigenen Wissens soll das analytische Instrumentarium der Wissenschafts- und Technikgeschichte erweitern und dazu beitragen, den produktiven Anteil der indigenen Völker Süd- und Mittelamerikas an der Geschichte des Kautschuks besser aufzuarbeiten. Dieser Ansatz ist in der aktuellen Wissenschafts- und Technikforschung keineswegs isoliert, vielmehr wird auch in anderen Themenfeldern die Bedeutung nichtwissenschaftlichen Wissens verstärkt untersucht. ${ }^{12}$

10 Nancy Paine Norton, Industrial Pioneer. The Goodyear Metallic Rubber Shoe Company, Unpublished Doctor-Thesis, Cambridge, Mass. 1950, S. 1.

11 Siehe insb. Erland Nordenskiöld, Om Indianernes Anvendelse af Gummi i Sydamerika, in: Geografisk Tidsskrift 24, 1918, S. 80-86. Ders., The American Indian as an Inventor, in: The Journal of the Royal Anthropological Institute of Great Britain and Ireland 59, 1929, S. 273-309. Dorothy Hosler, Sandra Burkett u. Michael J. Tarkanian, Prehistoric Polymers. Rubber Processing in Ancient Mesoamerica, in: Science 284, 1999, S. 1988-1991; mit Abbildungen: Michael J. Tarkanian u. Dorothy Hosler, An Ancient Tradition Continues: Modern Rubber Processing in Mexiko, in: E. Michael Whittington, The Sport of Life and Death: The Mesoamerican Ballgame, London 2001, S. 116-121; Michael R. Dove, The Life-Cycle of Indigenous Knowledge, and the Case of Natural Rubber Production, in: Roy Ellen, Peter Parkes u. Alan Bicker, Indigenous Environmental Knowledge and its Transformations, London, New York 2003, S. 213-251 (bezieht sich auf ,smallholder cultivators of Pará rubber in Southeast Asia“" (ebd., S. 214).

12 Siehe Fritz Böhle, Annegret Bolte, Ingrid Drexel u. Sabine Weishaupt, Grenzen wissenschaftlich-technischer Rationalität und ,anderes Wissen', in: Ulrich Beck u. Wolfgang Bonß (Hg.), Die Modernisierung der Moderne, Frankfurt a.M. 2001, S. 96-105; Matthias 
Der Begriff des traditionellen indigenen Wissens wird zudem in der internationalen Politik und im internationalen Recht etwa seit Mitte der 1980er Jahre verwandt. ${ }^{13}$ Eine einheitliche Definition hat sich in der Literatur bisher nicht durchgesetzt. ${ }^{14}$ Der Begriff bezeichnet ein durch seine Träger und Schöpfer selbst nicht schriftlich kodifiziertes Wissen, das indigene Völker Amerikas in Bezug auf Materialien, Pflanzen, Tiere, technische Prozesse, Produkte und Naturphänomene haben. Dieses Wissen ist als solches unsichtbar, kann aber anhand von Indizien erschlossen werden. Als solche Indizien kommen auch Handlungen infrage, wenn durch sie Probleme gelöst werden. Ein Automechaniker, der ein Auto repariert, versteht offenbar etwas davon, auch wenn er auf Fragen keine Antwort gibt. Analog haben auch Indianer offenbar Wissen über den Stoff Kautschuk, auch wenn sie dieses Wissen in den verfügbaren Quellen nicht direkt artikulieren.

Indigenes Wissen wird meist als traditionelles Wissen bezeichnet, eine unglückliche Bezeichnung, weil das indigene Wissen dadurch etwas Statisches erhält. ${ }^{15}$ „Traditionelles Wissen“ scheint ein für alle Mal festzuliegen. Das indigene Wissen rückt auf diese Weise in die Nähe des angeborenen Wissens von Tieren, das auch ,immer schon“ da ist. Es ist aber davon auszugehen, dass auch indigenes Wissen dynamisch ist und immer wieder durch individuelle Entdeckungen und Erfindungen transformiert wird, die dann von anderen übernommen wurden (wie bereits Tarde postulierte). ${ }^{16}$ Auf der anderen Seite wird der Begriff des indigenen Wissens gern mystifiziert und dem ,positivistischen westlichen Wissen entgegengesetzt. Es sei ,ökologischer' und ,im Einklang mit der Natur'. Dieses verbreitete Stereotyp wird heute kritisiert. Vorsichtig sollte man auch sein, indigenes Wissen mit einem Begriff von Michael Polanyi insgesamt als ,tacit knowledge' zu kategorisieren. Vielmehr ist anzunehmen, dass es ähnlich wie unser Wissen auch, beträchtliche explizite Anteile hat und zumindest in wichtigen Stücken durch Lehren und Lernen übertragen

Heymann u. Ulrich Wengenroth, Die Bedeutung von 'tacit knowledge' bei der Gestaltung von Technik, in ebd., S. 106-121; Frank Uekötter, Die Wahrheit ist auf dem Feld. Eine Wissensgeschichte der deutschen Landwirtschaft, Göttingen 2010, S. 26f.; sowie Philipp Sarasin, Was ist Wissensgeschichte?, in: Internationales Archiv für Sozialgeschichte der Literatur 36, 2011, S. 159-172.

13 Eine gute Übersicht bei Manuela Carneiro da Cunha, ,Culture' and Culture. Traditional Knowledge and Intellectual Rights, Chicago 2009, S. 10-26.

14 Xuan Li, Overcoming Market Failure and Rationalizing Traditional Indigenous Medicinal Knowledge Protection Regimes. An Economic Approach and Case Study in China, Bamberg 2007, S. 16f.; siehe auch Arne Kalland, Indigenous Knowledge - Prospects and Limitations, in: Ellen et al. (wie Anm. 11), S. 319-335, hier S. 321f.

15 Zur Kritik vgl. Carneiro da Cunha (wie Anm. 13), S. 28; siehe auch Arin Agrawal, Dismantling the Divide Between Indigenous and Scientific Knowledge, in: Development and Change 26, 1995, S. 413-439; siehe auch Roy Ellen u. Holly Harris, Introduction, in: Ellen et al. (wie Anm. 11), S. 1-29.

16 Vgl. Gabriel Tarde, Die Gesetze der Nachahmung (Les lois de l'imitiation, Paris 1890), Frankfurt a.M. 2009, S. 110-129. 
wird. ,Tacit ' ist es allerdings weitgehend für die wissenschaftshistorische und technikhistorische Literatur zum Kautschuk, die dieses Wissen ignoriert. ${ }^{17}$

Indigenes Wissen dürfte prinzipiell ähnlich entstehen wie westliches technisches Wissen, nämlich durch Beobachtung, Ausprobieren, logisches Schließen, Analogiebildung, Zufall. Man kann z.B. annehmen, dass die Beobachtung des Verhaltens von Tieren ${ }^{18}$ ebenso bedeutend für die Wissensentstehung ist wie die Übertragung von praktischen Erkenntnissen von einem Technikbereich auf einen anderen. ${ }^{19}$

Worin unterscheidet sich das indigene Wissen vom wissenschaftlichen Kautschukwissen? Oft werden die ethnomethodologischen Laborstudien etwa von Bruno Latour so interpretiert, dass diese erwiesen hätten, dass auch wissenschaftliches Wissen in hohem Grade kontextabhängig und keineswegs universal sei. ${ }^{20}$ Diese Ergebnisse dürfen aber nicht dahin missverstanden werden, als gebe es gar keinen Unterschied zwischen indigenem Wissen und wissenschaftlichem Wissen.

Aus dem fundamentalen Unterschied, dass indigenes Wissen nicht schriftlich organisiert ist, lassen sich vielmehr einige Unterschiede ableiten, die man nicht nivellieren sollte. Es gibt nur sehr wenige moderne ethnologische Feldstudien zu indigenem technischen Wissen ${ }^{21}$ und keine solche Studie wurde bislang zur indigenen Kautschukbearbeitung durchgeführt. Sicher ist aber indigenes Wissen nicht theorieförmig organisiert oder logisch durchstrukturiert, weil wissenschaftliche Theorie Schrift voraussetzt. Es wird eher situationsgebunden sein als abstrakt, eher additiv als subordinativ und möglicherweise auch in Mythen organisiert. ${ }^{22}$ Es ist zwar nicht so statisch, wie der Begriff

17 Vgl. zur Kritik an Konstrukten eines ,ökologischen“ indigenen Wissens etwa Anne-Christine Hornborg, Mi'kmaq Landscapes. From Animism to Sacred Ecology, Aldershot 2008, S. 14-30 und passim. Zum ,tacit knowledge' Michael Polanyi, Implizites Wissen, Frankfurt a.M. 1985; sowie zuletzt Harry Collins, Tacit and Explicit Knowledge, Chicago 2010.

18 Vgl. mit Beispielen Jens Soentgen u. Klaus Hilbert, Präkolumbianische Chemie, in: Chemie in unserer Zeit 46, 2012, S. 322-334; siehe auch Paul Newton u. Nathan Wolfe, Can Animals Teach us Medicine?, in: British Medical Journal 305, 1992, S. 1517-1518. In Indianermythen sind es oft Tiere, die den Menschen bestimmte Technologien vermitteln.

19 Für das Beispiel Rad-Töpferscheibe vgl. André Leroi-Gourhan, Milieu et technique, Paris 2002 (1945/1973), S. 344-345, passim. Leroi-Gourhan hat seine Erkenntnisse nicht auf den Kautschuk angewandt, seine Zeilen zu dieser Substanz bleiben wenig ergiebig; vgl. ders., L'homme et la matière, Paris 2004 (1943/1971), S. 232f.

20 Kavita S. Philip, Indigenous Knowledge. Science and Technology Studies, in: International Encyclopedia of the Social \& Behavioral Sciences, Elsevier 2001, S. 7292-7297, hier S. $7292 \mathrm{f}$.

21 Zur Keramik siehe die ausgezeichnete Studie von Fabiola Andrea Silva, As tecnologias e seus significados. Um estudo da cerâmica dos Asuriní do Xingu e da cestaria dos KayapoXikrin sob uma perspectiva etnoarqueologica. Faculdade de Filosofia e Ciências Humanas da Universidade de São Paulo, São Paulo 2000.

$22 \mathrm{Zu}$ diesen und weiteren Kennzeichen mündlichen Wissens siehe Rainer Totzke, BuchstabenFolgen. Schriftlichkeit, Wissenschaft und Heideggers Kritik an der Wissenschaftsideologie, Weilerswist 2004, S. 36-46 mit weiterer Literatur. 
„traditionell“" suggeriert, doch wird es andererseits auch nicht so dynamisch sein wie das europäische Wissen, das seine Dynamik, wie vielfach festgestellt wurde, seiner Schriftlichkeit verdankt. ${ }^{23}$ Sowenig wie komplexe symphonische Musik ohne musikalische Notationssysteme möglich ist, sowenig ist ohne Schrift Theorie möglich.

Wir wissen kaum, welche indigenen Gemeinschaften genau die Träger jenes Wissens waren, und welche Gemeinschaften es heute noch im Amazonasgebiet pflegen und weitergeben. Aus den Quellen entnehmen wir nur, dass besonders die heute ausgerotteten Omagua kompetente Verarbeiter von Kautschuk waren.

Nach der klassischen europäischen Auffassung war solches Wissen Gemeingut, konnte also von jedem, insbesondere auch von Europäern, ohne Weiteres genutzt werden. Erst heute wird, koordiniert durch die World Intellectual Property Organization, eine UN-Sonderorganisation mit Sitz in Genf, an Regeln für den Umgang mit solchem Wissen gearbeitet. Dabei werden aus den allgemeinen Prinzipien des geistigen Eigentums Grundsätze für den Zugang zu indigenem Wissen abgeleitet, wie der Prior Informed Consent (PIC), wonach Informanden, die über indigenes Wissen verfügen, sich vorher einverstanden erklären müssen, ehe ihr Wissen abgefragt wird. Ein anderes von der WIPO anerkanntes Prinzip ist das Equitable benefit-sharing. Dieses sieht vor, dass diejenigen, welche traditionelles Wissen zur Verfügung stellten, auch angemessen an den kommerziellen Gewinnen oder ideellen Vorteilen, wie Forschungsergebnissen, zu beteiligen sind. Diese Prinzipien liegen auch der Biodiversitätskonvention von 1992 zugrunde, welche den Zugang zu genetischen Ressourcen und den entsprechenden gerechten Vorteilsausgleich regelt.

Im Folgenden geht es nicht um die Rechte, die nach neuerer Auffassung bei der Nutzung indigenen Wissens zu beachten sind oder um die Frage, ob solche Rechte auch in der Vergangenheit schon hätten beachtet werden müssen. Dies wäre ein zweiter Schritt. In dieser Untersuchung steht das kautschukbezogene indigene Wissen im Fokus.

Dieses muss mindestens folgende Elemente haben: Zum einen gibt es eine botanische und biogeographische Komponente indigenen Kautschukwissens. Die Indianer wussten, welche Pflanzen zu welchen Zeiten Latex führten, wo man diese finden kann und wie man den Latex zapfen kann, ohne den Baum zu schädigen. Zudem war ihnen bekannt, wie man die Milch mit einem bestimmten Räucherverfahren so behandeln kann, dass daraus gefertigte Gegenstände stabil blieben und nicht in Fäulnis übergingen oder in der Hitze klebrig wurden. Dies ist die Komponente des Prozesswissens, man könnte auch von der chemischen Komponente sprechen, weil es sich dabei um eine Stofftransformation handelt. Schließlich verfügten die indigenen Völker über Produktwissen, konnten also die besonderen Eigenschaften des Stoffes

23 Vgl. ebd., S. 79-85 mit weiterer Literatur. 
in Gebrauchswerte umsetzen. Weitere Dimensionen sind denkbar (z.B. mythische oder historische Dimensionen), können jedoch mithilfe der von mir herangezogenen Quellen nicht rekonstruiert werden.

Entscheidend ist das Prozesswissen, die Kenntnis eines Prozesses, mit dem sich die Kautschukprodukte fertigen und gegen Degradierung konservieren lassen. Diese Methode ist das Kernstück indigenen Kautschukwissens, weil ohne sie die gefertigten Kautschukprodukte sehr kurzlebig und damit praktisch wertlos geblieben wären.

Man kann sofort den Einwand erheben, dass Begriffe wie „Wissen“ oder gar Prädikate wie „chemisch“ oder „,botanisch“ europäischen Ursprungs sind und dass sich mit solchen Begriffen indianisches Weltverständnis nicht angemessen rekonstruieren lässt. Es ist in der Tat vielfach nachgewiesen, dass das Weltverständnis der Völker Süd- und Mittelamerikas sich vom europäischen unterscheidet (und schon in Europa darf kein Kontinuum des Weltverständnisses vorausgesetzt werden). ${ }^{24}$

Die Gegenargumente liegen auf der Hand. Man könnte den Einwand polemisch kontern und feststellen, dass die Frage, ob man den indigenen Völkern Süd- und Mittelamerikas wirklich Wissen zusprechen kann, in einer Linie mit der älteren, im 16. Jahrhundert gewälzten Frage steht, ob diese Indianer überhaupt Menschen sind. Von solcher Polemik müssen wir keinen Gebrauch machen. Und doch gilt es zu betonen, dass man, wenn man aus methodischen Bedenken heraus Begriffe wie indigenes (chemisches, technisches, botanisches) Wissen aus der Kautschukgeschichtsschreibung verbannt, zugleich die Möglichkeit verbaut, die Indianer in dieser Geschichte als technisch begabte, erfinderische Menschen zu thematisieren. Dieses Gegenargument möchte ich ergänzen durch eine forschungspragmatische Überlegung. Es besteht gar keine andere Möglichkeit, zu einem vertieften Verständnis der Rolle indigenen Wissens in der Geschichte des Kautschuks zu kommen, als von unseren europäischen Begriffen auszugehen. Diese werden sich dann im Forschungsprozess jedoch weiterentwickeln.

Die Situation bei der Wissensgeschichte des Kautschuks ähnelt der bei der grammatischen Rekonstruktion der Indianersprachen. Hier wurde auch bereits frühzeitig festgestellt, dass die am Beispiel der griechischen und lateinischen Grammatik gebildeten europäischen Begriffe nicht recht passen, weil diese Sprachen von Grund auf anders gebaut sind. ${ }^{25}$ Schon die Aufzeichnung der gesprochenen Indianersprachen mithilfe des lateinischen Alphabets erwies

24 Siehe z.B. Eduardo Viveiros de Castro, Cosmological Deixis and Amerindian Perspectivism, in: The Journal of the Royal Anthropological Institute 4, 1998, S. 469-488; ähnlich bereits Fritz Krause, Maske und Ahnenfigur, in: Ethnologische Studien 1, 1931, S. 344-364. Ein davon leicht abweichendes Verständnis amazonischer Weltdeutung vertritt Philipp Descola, Jenseits von Natur und Kultur, Frankfurt a.M. 2011, S. 197-218.

25 Georg Friederici, Amerikanisches Wörterbuch und Hilfswörterbuch für den Amerikanisten. Deutsch - Spanisch - Englisch, 2. Auflage, Hamburg 1960, S. 25. 
sich als nahezu unmöglich. ${ }^{26}$ Trotz dieser Schwierigkeiten hat man nicht darauf verzichtet, die Indianersprachen aufzuzeichnen und grammatisch zu rekonstruieren. Dabei wurden dann neue Zeichen und neue Begriffe eingeführt. Analog kann man auch bei der Rekonstruktion indigenen chemischen und technischen Wissens hoffen, dass unpassende Begriffe mit dem Fortschritt der Forschung ersetzt werden.

Um die Überlegungen zusammenzufassen: Das Gewicht der methodischen Bedenken ist beträchtlich, darf aber nicht dazu führen, dass der Versuch, die Bedeutung indigenen Wissens für die Geschichte des Kautschuks zu rekonstruieren, aufgegeben wird, ehe er auch nur begonnen wurde. Denn wenn die kognitiven Leistungen der indigenen Völker Süd- und Mittelamerikas aus Sorge um methodische Probleme nicht rekonstruiert werden, entstehen Kautschukgeschichten, die ausschließlich die Bedeutung europäischer und nordamerikanischer Wissenschaft und Technik betonen (um nicht einzelne Autoren zu diffamieren, sei auf Belege an dieser Stelle verzichtet). Die Indios kommen in diesen Geschichten dann nur als ,Naturvölker', die poetische Namen erfinden (,tränendes Holz") oder als Opfer grausamer Ausbeutung und Verfolgung vor, aber nicht als kompetente Akteure. Solche Kautschukhistorien sind aber dem Verdacht, eurozentrisch zu sein oder gar kolonialistische Perspektiven fortzusetzen, relativ wehrlos ausgeliefert.

Wir können das indigene Wissen nur indirekt rekonstruieren. Denn leider gibt es weder moderne ethnologische Studien noch Beschreibungen indigenen Kautschukwissens aus erster Hand (wie es sie für das Wissen indigener Völker Nordamerikas sehr wohl gibt). ${ }^{27}$ Daher muss zum einen auf indigene Kautschukartefakte in Museen, zum anderen auf Beschreibungen von Europäern, die indigene Kautschukprodukte und Produktionsmethoden beobachtet haben, zurückgegriffen werden. Das letztere wird hier unternommen, indem die verfügbaren Augenzeugenberichte, sei es von Entdeckern, von Missionaren, von Conquistadoren oder, später, von Reisenden und Naturforschern gesichtet und ausgewertet wurden. Viele der oben und im Folgenden zitierten Quellen werden dabei erstmals für die Darstellung der Geschichte des Kautschuks herangezogen. Der Schwerpunkt liegt auf Quellen über die Kautschuknutzung im Amazonasbecken. Dort ist die Heimat der Hevea brasiliensis, der wichtigsten latexliefernden Pflanze, dort lässt sich eine hochdifferenzierte indigene Kautschuktechnologie nachweisen. Die Quellen über die Kautschuktechnologie Mittelamerikas bedürften einer separaten Untersuchung.

26 Vgl. Franz Xavier Veigl, Gründliche Nachrichten über die Verfassung der Landschaft von Maynas, in Süd-Amerika, bis zum Jahre 1768, beschrieben von Franz Xavier Veigl, in besagter Provinz vormaligem Missionar der Gesellschaft Jesu, in: Christoph Gottlieb von Murr (Hg.), Reisen einiger Missionarien der Gesellschaft Jesu in Amerika. Aus ihren eigenen Aufsätzen, Nürnberg 1785, S. 130f.

27 Vgl. Wendy Makoons Geniusz, Our Knowledge is not Primitive. Decolonizing Botanical Anishinaabe Teachings, Syracuse u. New York 2009. 


\section{Pflanzliche Herkunft und Etymologie}

Es gibt etwa 2.000 latexliefernde Pflanzen, ${ }^{28}$ darunter viele in der Alten Welt. Latex liefert zum Beispiel die Zypressen-Wolfsmilch, Euphorbia cyparassias, die Gänsedistel, Sonchus oleraceus oder der Ruten-Lattich, Lactuca viminea. Diese Pflanzen enthalten zwar, auf die Trockensubstanz berechnet, sogar mehr Kautschuk als Hevea brasiliensis, absolut aber sind es bescheidene Mengen. ${ }^{29}$ Jedoch kommen in der Alten Welt auch kautschukführende Bäume vor, wie der als Zimmerpflanze heute beliebte Ficus elastica, der aus Indien stammt. In China und in Afrika kommen ebenfalls Pflanzen vor, die reichlich Latex liefern. Dennoch wurde dieses Potenzial offenbar nicht systematisch genutzt. Zwar wird gelegentlich behauptet, dass in China vor Bekanntwerden der südamerikanischen Kautschukprodukte bereits Kautschuk produziert wurde doch lassen sich diese Aussagen anhand der Quellen nicht erhärten, es scheint sich bei den damals aus China gelieferten elastischen, farbigen Kugeln nicht um eigentlichen Kautschuk, sondern um ein Surrogat gehandelt zu haben. ${ }^{30}$ Die Nutzung einer latexführenden Pflanze im Südwesten Madagaskars ist ebenfalls bezeugt, doch wurde der Saft lediglich für Fackeln genutzt ${ }^{31}$ (die Identifikation der Pflanze ist schwierig). ${ }^{32}$

Die botanischen Voraussetzungen der Entdeckung bzw. Erfindung des Kautschuks waren auch in der Alten Welt gegeben, wie später die Nutzung der Löwenzahnart Taraxacum kok-saghyz als Latexlieferant im Zweiten Weltkrieg zeigt. ${ }^{33}$ Kautschuk ist nicht nur eine Entdeckung, wie man etwa eine bisher unbekannte Pflanze oder ein Mineral entdecken kann, sondern auch eine Erfindung, weil die Nutzung des Materials den Aufbau von Wissen und die Erfindung neuartiger Formungs- und Materialbehandlungsmethoden voraussetzt. Zu Recht schreibt der schwedische Ethnologe Erland Nordenskiöld:

28 Dieter Heß, Pflanzenphysiologie, 11. Auflage, Stuttgart 2008, S. $166 f$.

29 Ludwig Diels, Ersatzstoffe aus dem Pflanzenreich, Stuttgart 1918, S. 303.

30 M. Bourgeois, Extrait d'une Lettre de M. Bourgeois, Missionnaire, Péking, le 19. Novembre 1784, in: Mémoires concernant Les Chinois, Tome XI, Paris 1786, S. 577-579. Georg Christoph Lichtenberg, Physikvorlesung, nach J. Chr. P. Erxebens Anfangsgründen der Naturlehre. Aus den Erinnerungen von Gottlieb Gamauf. Bearb. und mit einer Einleitung versehen von Fritz Krafft, Wiesbaden 2007, S. 34; die Erinnerungen wurden erstmals 1808-1812 publiziert; die Vorlesungen von Lichtenberg wurden in den 1790er Jahren gehalten. Rehmsche Buchhandlung, Die Botanik nach ihren neuesten Ansichten dargestellt. Zweyte Abtheylung, Wien 1815, S. 115f.; Lüdersdorff (wie Anm. 5), S. 14; Wu Zhengyi, Peter H. Raven u. Deyuan Hong, Flora of China. Vol. 5: Ulmaceae through Basellaceae, Beijing 2003, S. 42.

31 Alexis-Marie de Rochon, Des Abbe Rochon, Mitgliedes der Akademie der Wissenschaften zu Paris und Petersburg u. Reise nach Madagaskar und Ostindien usw., übersetzt von Georg Forster, Berlin 1792, S. 132.

32 Vgl. Victor Forbin, Le Caoutchouc dans le Monde, Paris 1943, S. 19-21.

33 Ulrich Giersch u. Ulrich Kubisch, Gummi. Die elastische Faszination, Ratingen, Berlin 1995, S. $155-157$. 
"It is a matter of fact that in pre-Columbian times the Indians were acquainted with all the qualities that make rubber so valuable in modern industry. No corresponding discovery had ever been made in the Old World prior to the discovery of America, in spite of the fact that both in Asia and Africa there are found rubber trees of various kinds. "34

In Mittel- und Südamerika gibt es mehrere latexführende Bäume, von denen Hevea brasiliensis, der im Amazonasgebiet vorkommt, der wichtigste ist. Die Etymologie des Wortes Cauchuc ${ }^{35}$ oder Cahuchu ${ }^{36}$ lässt sich nicht zweifelsfrei klären, auch nicht, welcher Indianersprache es ursprünglich entstammt. In der Literatur finden sich mehrere Etymologien. Am genauesten aus den Quellen begründet ist die Etymologie des Sprachforschers Georg Friederici, der Belege für die peruanische Herkunft des Wortes caucho sammelte und nachwies, dass es schon 1613 in einem Khetschua-Wörterbuch auftauchte, mit der Bedeutung ,Zauberer“ (encantador). ${ }^{37}$ Sehr populär, wenngleich unbewiesen, ist die Namensdeutung ,weinendes Holz“, die der Botaniker W.H. Johnson aufbrachte. ${ }^{38}$ Julius Platzmann nimmt eine Herkunft aus dem Tupí an und behauptet, Kautschuk bedeute „Baumharz“. 39

Als Kautschuk wird heute in der deutschen Fachliteratur meist das nichtvulkanisierte Latexkoagulat bezeichnet, mit Gummi ausschließlich mit Schwefel vulkanisierte Produkte. Das Wort Gummi (aus dem Griechischen kómmi $=$ Harz) wird dabei in seiner Bedeutung stark verengt. Der umgangssprachliche Gebrauch, an den auch ich im Folgenden anschließe, ist lockerer. In der älteren deutschen Literatur wird statt von Kautschuk auch von Federharz gesprochen, in Übersetzung der lateinischen Bezeichnung resina elastica. In den USA und in England hingegen spricht man unterschiedslos von (India) ,rubber', in Erinnerung an eine von Joseph Priestley popularisierte Entdeckung, die möglicherweise auf den Instrumentenbauer Nairne zurückgeht, ${ }^{40}$ dass sich mit Kautschukwürfeln Bleistiftzeichnungen besser wegreiben ließen als mit dem zuvor genutzten Brotteig. ,Cured rubber' meint den geräucherten Kautschuk; das aus der lateinischen Entsprechung abgeleitete Wort to cure bedeutet im Englischen sowohl heilen wie auch räuchern.

34 Nordenskiöld 1929 (wie Anm. 11), S. 279.

35 Cobo (wie Anm. 1), S. 268.

36 Charles Marie de la Condamine, Relation abrégé d'un Voyage fait dans l'interieur de L'Amerique Méridionale, Paris 1745, S. 78, mit dem Hinweis „prononcez: Cahout-chou“.

37 Georg Friederici, Lehnwörter exotischer Herkunft in europäischen Sprachen, in: Zeitschrift für französische Sprache und Literatur 58, 1938, S. 135-155, hier S. 145f. In Mexiko und Teilen Mittelamerikas spricht man statt von Kautschuk von Ule oder Hule.

38 William H. Johnson, The Cultivation and Preparation of Para Rubber, London 1909 (zuerst 1904), S. 1.

39 Julius Platzmann, Das anonyme Wörterbuch Tupi-Deutsch und Deutsch-Tupi. Leipzig 1901, XVII, vgl. auch S. 376. Das Wörterbuch baut auf einem 1795 in Lissabon erschienenen auf.

40 Joseph Priestley, A Familiar Introduction to the Theory and Practice of Perspective, London 1770, S. XV. 
In Brasilien spricht man von „borracha“, nach einem alten Wort für Flasche; der Kautschukbaum heißt dort pau-seringa, Spritzenbaum, und der Kautschukzapfer seringueiro, „Spritzenmann“, in Erinnerung an die Gummispritzen, deren Fertigung nach dem Zeugnis de la Condamines ${ }^{41}$ die in der Provinz Pará ansässigen Portugiesen von den (heute ausgerotteten) Omagua erlernt haben.

\section{Indigene Kautschukprodukte}

Gummiprodukte, vom Gummistiefel über den Regenmantel, vom Autoreifen bis zum Quietscheentchen sind Teil der modernen materiellen Kultur. Sehr viele dieser Gummiprodukte wurden zuerst von Indianern erfunden. Ihre Produktideen wurden von Europäern und Nordamerikanern übernommen und weiterentwickelt, wie anhand einiger Beispiele gezeigt wird.

Hochspringende Gummibälle waren es, welche, wie oben gezeigt, die Europäer zuerst mit den erstaunlichen Eigenschaften des Kautschuks bekannt machten. Das Material wurde aber weitaus vielfältiger und kreativer eingesetzt. Er wurde zum Beispiel zur Produktion von Klistieren und damit für Spritzen herangezogen. Dabei wurde mit dem Verfahren der verlorenen Form gearbeitet, indem das Produkt um einen Tonkern geformt wurde, der dann durch eine Öffnung ausgewaschen wurde. Dazu wurde ein sandiger Ton verwendet, der sich leicht wieder zerkleinern und hervorholen ließ. In die Flaschenöffnung wurde dann ein hohler Vogelknochen gesteckt, der als Kanüle diente. Auf diese Weise wurden Klistiere produziert, die etwa, wie u.a. der französische Naturforscher de la Condamine von den Omagua berichtet, vor Festgelagen herumgereicht wurden..$^{42}$ Auch halluzinogene Substanzen wurden durch solche Klistiere eingeführt; ${ }^{43}$ kleinere Spritzen dienten dem Schnupfen halluzinogener Substanzen durch die Nase. ${ }^{44}$ Franz Veigl schreibt:

„Das Mannsvolk (der Omagua) bildet aus gewissem ganz besonderen Harze, auf peruanisch Cauchu, (Kautschu) einige hohle Kürbschen, mit an die Mündung angefügten dünnen Röhrlein. Diese Plutzergen oder Kürbschen, bleiben immer sehr biegsam, und wenn man sie so zusammen drücket, daß die Luft völlig heraus muß, das Röhrlein aber sodann in etwas flüßiges hinein hält, säuft sich damit das Plutzergen von selbst ganz voll an. Drücket man es alsdann wieder mit der Hand, so läuft das eingesogene nach Maas der drückenden Gewalt, gleich einer Spritze, sehr weit hinaus." ${ }^{\text {"45 }}$

Die Originalität dieser Spritzen gegenüber den mit Stempel arbeitenden europäischen Äquivalenten ist allen Reisenden, die darüber berichtet haben,

41 De la Condamine (wie Anm. 36), S. 79.

42 Ebd., S. 79f.

43 Oskar Hovorka u. Adolf Kronfeld, Vergleichende Volksmedizin, Bd. 1, Stuttgart 1908, S. 237.

44 Veigl (wie Anm. 26), S. 87.

45 Ebd., S. 86f. 


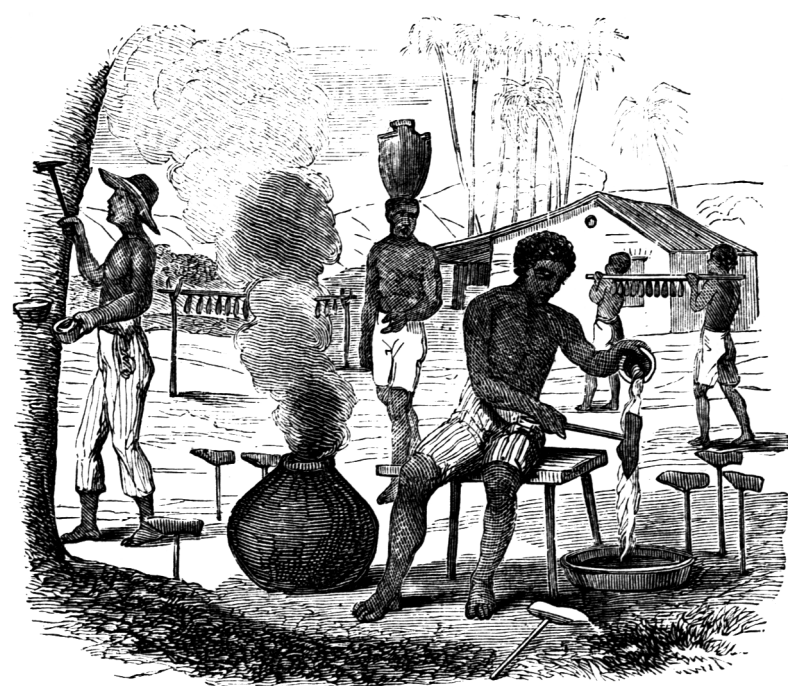

Abb. 1: Eine zeitgenössische Darstellung der indigenen Kautschukverarbeitung Anfang des 19. Jahrhunderts in Pará. Quelle: D.P. Kidder u. J.C. Fletcher, Brazil and the Brazilians, London 1857, S. 553.

bewusst. Kautschukklistiere haben den Vorzug, dass sie Selbstbehandlung zulassen, während dies bei Spritzen mit Stempeln nicht so leicht möglich ist. ${ }^{46}$ Die Spritzen regten die Europäer zu eigenen Medizinprodukten an, zu Handpumpen für Injektionen, Spülungen, Milchpumpen usw. ${ }^{47}$ Auch heute noch sind solche Gummiprodukte in Gebrauch. Es gab weitere medizinische Anwendungen, die modern wirken. Kautschuklamellen wurden von den Couna in der Provinz Darién an der Grenze Kolumbiens als Beißschiene genutzt, die Fieberkranken zwischen die Zähne geschoben wurde, damit sie diese nicht in ihren Krämpfen zerknirschen. ${ }^{48}$ Der Milchsaft scheint zudem vielfältig innerlich angewandt worden zu sein, wie schon Cobo mitteilt, unter anderem zur Behandlung von Darmblutungen („curar cámaras de sangre“). ${ }^{49}$

Von der Kautschukflasche zum hohlen Ball (und umgekehrt) ist es nur ein kleiner Schritt, da lediglich die Öffnung verschlossen werden muss. ${ }^{50}$ Das Ballspiel mit hohlem oder massivem Ball war nicht nur den Maya oder Azteken bekannt, sondern wurde im ganzen tropischen Südamerika geübt, wie das Studium alter Reiseberichte zeigt. ${ }^{51}$

46 Johann Georg Krünitz, Oekonomische Encyklopaedie oder allgemeines System der Staats-, Stadt-, Haus- u. Landwirthschaft, und der Kunst-Geschichte, Bd. 22, 1789, S. 82f. Online unter: http://www.kruenitz1.uni-trier.de/ (Stand 27.11.2013), Artikel Harz, Feder-Harz.

47 Ebd., S. 83.

48 Forbin (wie Anm. 32), S. 12.

49 Cobo (wie Anm. 1), S. 269.

50 Nordenskiöld 1918 (wie Anm. 11), S. 85.

51 Theodore Roosevelt, Through the Brazilian Wilderness and Papers on Natural History, New York 1926, S. 159. Weitere Beschreibungen hat Nordenskiöld 1918 (wie Anm. 11), 
Ferner wurden Kautschukbänder hergestellt, mit denen verschiedene Objekte durch Umwickeln wasserdicht gemacht wurden, ${ }^{52}$ es wurden Schuhe gefertigt, wasserdichte, gummibeschichtete Textilien und Flaschen. ${ }^{53}$ Alle diese Produkte sind heute noch in Gebrauch.

Die Indianer nutzten aus Kautschuk gefertigte Ringe als Schmuck und zur Bündelung von Ästen usw. Gummiringe sind heute in jedem Supermarkt, in jedem Schreibwarenladen erhältlich und wohlbekannte Begleiter des Alltags. Cornelius Pauw schreibt in seinen Recherches Philosophiques zudem, dass solche Ringe bei manchen indigenen Völkern auch als Penisringe zur Luststeigerung verwandt wurden. ${ }^{54}$

Nicht zuletzt wurde Kautschuk für Spielzeugpuppen verwandt. Der Latex wurde hierfür in Formen gegossen; auch hohle Spielpuppen wurden gefertigt, wie Krünitz berichtet:

„Übrigens werden uns noch verschiedene andere Geräthe und Körper von diesem Harze, auch oft unförmliche Figuren von allerley Thieren, Bälle etc. über Spanien aus Amerika zugeführt, welche Sachen gemeiniglich, die Bälle ausgenommen, hohl sind. Fast alles ist mit Laubwerk, oder andern Figuren, die man darauf eindrückt, wenn die Materie noch weich ist, aber nicht sehr mit Geschmack, geziert. “55

Ferner wurde Kautschuk als Zunder und für nichttropfende, doch aufgrund der Rußpartikel hellleuchtende Fackeln verwendet. ${ }^{56}$ Die Jivaros nutzten den Kautschuk auch als Brandsatz in der Kriegsführung, indem sie Stücke entzündeten Kautschuks beim Angriff auf Dächer warfen. ${ }^{57}$

Auch wenn die Indianer weder den Radiergummi noch den Autoreifen erfunden haben, zeigt dieser Überblick, dass ein beträchtlicher Teil moderner Gummiprodukte bereits von den Indianern erdacht und von den Europäern, nachdem sie die indianischen Produkte durch den Übersee-Handel kennenlernten, adaptiert wurden.

S. 80-84 zusammengetragen. Vgl. insbesondere die Beschreibung von Joseph Gumilla, El Orinoco Ilustrado y Defendido, Historia Natural, Civil, Y Geographica De este Gran Rio Y de Sus Caudalosas Vertientes, Madrid 1745, S. 190f. Ein Foto des Kopfballspieles bei den Paressi-Kabisi zeigt Max Schmidt, Die Paressi-Kabisi, in: Baessler-Archiv 4, 1914, S. 183.

52 Carl Friedrich Philipp v. Martius, Beiträge zur Ethnographie und Sprachenkunde Amerikas zumal Brasiliens. Bd. I: Zur Ethnographie, Leipzig 1867, S. 440.

53 Z.B. Pierre Barrere, Nouvelle Relation de la France Equinoxiale, Paris 1743, S. 139-141.

54 Cornelius de Pauw, Recherches Philosophiques sur Les Américains, Berlin 1777, S. 54.

55 Krünitz (wie Anm. 46), S. 82.

56 Z.B. Martius (wie Anm. 52), S. 440; Marquis de Wavrin, Les Jivaros. Réducteurs de Têtes. Récit d'Exploration Publié par Gaston Bunnens, Paris 1941, S. 179. Der Gebrauch von Kautschuk zu Beleuchtungszwecken wurde schon auf der Zweiten Fahrt des Columbus auf Hispaniola beobachtet; vgl. den Brief des Michele de Cuneo (verfasst 1495), in: Luigi Firpo, Colombo - Vespucci Verazzano, Turin 1966, S. 47-76, hier S. 60.

57 Wavrin (wie Anm. 56), S. 103. 
Einige der hier genannten indigenen Kautschukprodukte sind so originell, dass man von Erfindungen sprechen muss. Das gilt insbesondere für die Kautschukspritzen, die mit einem anderen Material überhaupt nicht funktionieren würden. Diese Spritzen erreichen nicht nur ein bekanntes technisches Ziel auf neuem Wege, sondern verwirklichen ein neues Ziel mit neuen Mitteln. Andere Kautschukprodukte, wie die Schuhe, die Gummiflaschen oder die Ringe erreichen ein bekanntes Ziel mit neuen Mitteln. Erst die von den Indianern entwickelte Vielfalt der Anwendungen, von denen wohl nur ein Teil den Europäern bekannt wurde, machte den Stoff in der alten Welt interessant. Alle diese Kautschuknutzungen setzten aber voraus, dass man in der Lage ist, eine Flüssigkeit gezielt in einen geformten Feststoff zu verwandeln und dass man die Eigenschaften dieses Stoffes so kontrolliert, dass dieser längere Zeit stabil bleibt und nicht zu faulen beginnt oder in der Hitze klebrig wird. Die Kontrolle über den heiklen Stoff, die gezielte Transformation des flüssigen Latex in geformte und u.U. hohle Dinge und die Stabilisierung seiner erwünschten Eigenschaften sind die zentralen Errungenschaften der indigenen Kautschuktechnologie.

\section{Probleme des unbehandelten Kautschuks}

Latex, der aus einem verwundeten Hevea brasiliensis-Baum rinnt, kann man trocknen lassen, man erhält auf diese Weise Gebilde, die zwar etwas klebrig sind, aber durchaus alle Eigenschaften normaler Gummiprodukte aufweisen. Doch Kautschuk, der nur getrocknet wurde, weist Probleme auf, die vor allem der deutsche Gummipionier Lüdersdorff sehr plastisch schilderte, und die jeder kennt, der mit Gummi umgeht. Setzt man ein Kautschukprodukt dem Tageslicht aus,

„so beginnt nach kurzer Zeit die Zersetzung. An den dünnsten Stellen wird das Federharz zuerst angegriffen; es nimmt die Eindrücke der Finger an, und die Linien der Haut bleiben dauernd darauf sichtbar; dehnt man dasselbe aus, so reißt es bald [...]. Dieser Zustand der Veränderung nimmt immer mehr zu, und erstreckt sich bald durch die ganze Masse, die nun in das zweite Stadium übergeht. Dies ist ein völliges Klebrigwerden, welches mehr und mehr überhand nimmt, und die Masse in einen Zustand des Zerfließens bringt [...]. Das dritte und letzte Stadium ist endlich ein allmähliches, anfänglich oberflächliches, abermaliges Trockenwerden, welches durch Bildung einer harten Haut entsteht, die in ihrer Dicke immer mehr zunimmt [...] jetzt ist die Zersetzung beendigt. Das Federharz ist nun eben so spröde und brüchig, als es früher biegsam und elastisch war.“58

58 Lüdersdorff (wie Anm. 5), S. 34f. 
Zudem ist unbehandelter Kautschuk auch sehr empfindlich gegen mikrobiellen Befall, Kautschukwaren konnten regelrecht verfaulen, wie ein amerikanischer Kautschukhistoriker anschaulich darstellt:

„It was during the winter of 1832 that Goodyear passed the New York store of the Roxbury Company. Wearing a ragged coat, and a blacksmith's leather apron for additional warmth, he made his momentous visit to the rubber works, and met Proprietor Chaffee. Chaffee welcomed him, and confided the bad news that his goods were going, sour'. It was true. Rubberized cloth was literally rotting in warehouses: Chaffee had actually buried $\$ 20,000$ worth of it to keep it off the market. " ${ }^{\text {59 }}$

Die wichtigste moderne Methode, mit diesen Problemen umzugehen, besteht darin, Schwefel mit Kautschuk reagieren zu lassen. Der Einsatz von Schwefel wurde zuerst von Friedrich Lüdersdorff 1832 ausführlich publiziert; Lüdersdorff ließ zum einen eine Lösung von Schwefel in Terpentinöl auf Kautschuk wirken ${ }^{60}$ empfahl andererseits das Verreiben von Schwefelblüte auf klebrigen Kautschukstellen. ${ }^{61}$ Seine Idee wurde durch den siebten Band des ins Deutsche und ins Französische übersetzten und weit verbreiteten Lehrbuches der Chemie von Jöns Jakob Berzelius international bekannt gemacht. ${ }^{62}$ Der Einsatz von Schwefel wurde von Nathaniel Hayward nach seiner eigenen Aussage selbstständig entdeckt ${ }^{63}$ und 1839 auf Anregung von Goodyear patentiert. Goodyear übernahm das Patent von Hayward und entwickelte es später weiter. Die Priorität von Lüdersdorffs Entdeckung der Wirksamkeit von Schwefel gegenüber Hayward war in der anglo-amerikanischen Fachliteratur Mitte des 19. Jahrhunderts bekannt und wurde anerkannt, ${ }^{64}$ wobei man zugleich auf der Unabhängigkeit der Haywardschen Entdeckung bestand. In neueren englischsprachigen Werken zur Gummigeschichte wird Lüdersdorff nicht mehr erwähnt.

Der Schwefeleinsatz wurde von Charles Goodyear übernommen, indem er Haywards Patent kaufte und das Verfahren wiederum weiterentwickelte:

59 Charles M. Wilson, Trees and Test Tubes. The Story of Rubber, New York 1943, S. 44, vgl. auch S. 46.

60 Detailliert beschrieben in Lüdersdorff (wie Anm. 5), S. 45-53.

61 Ebd., S. 62.

62 Jöns Jakob Berzelius, Lehrbuch der Chemie, Bd. VII, 4. Auflage, Dresden u. Leipzig 1838, S. 106f. Zu plausiblen Indizien, dass der amerikanische Erfinder Hayward das Lüdersdorffsche Verfahren kannte, siehe Leo Eck, Friedrich Wilhelm Lüdersdorff, in: Gummi-Zeitung 32, 1932, S. 967-968, hier S. 968.

63 Hayward selbst datiert seine Entdeckung, dass Schwefel entscheidende Wirkung auf Kautschuk hat, auf das Jahr 1837, vgl. Nathaniel Hayward, Some Account of Nathaniel Hayward's Experiments with India Rubber, which Resulted in Discovering the Invaluable Compound of That Article With Sulphur, Norwich, Conn. 1865, S. 7.

64 Vgl. O.V., The Invention of Vulcanized India Rubber, in: Scientific American 31, 1857, S. 173; William H. Richardson Jr., The Boot and Shoe Manufacturers Assistant and Guide. Containing a Brief History of the Trade, Boston 1858, S. 109-111. 
Er erwärmte die Kautschuk-Schwefel-Mischung. Durch Kombination des Schwefeleinsatzes mit der von Thomas Hancock erfundenen Mastizierwalze ${ }^{65}$ wurde dann ein Verfahren entwickelt, das in seinen Grundzügen auch heute noch verwandt wird. Die Bezeichnung „Vulkanisation“, die William Brockedon prägte, und die dann von Hancock und schließlich auch von Goodyear übernommen wurde, ${ }^{66}$ suggeriert eine nicht nur graduelle qualitative Verbesserung, sondern eine abrupte Wesensveränderung, die zu einem universell einsetzbarem Material führte, das anschließend seinen ,Siegeszug um die Welt' angetreten hätte. Goodyear selbst nutzte 1839 noch die Bezeichnung „metallic gum-elastic“. ${ }^{67}$

Durch die von Lüdersdorff entdeckte Behandlung mit gelöstem oder pulverförmigem Schwefel, die von den amerikanischen Erfindern Hayward und Goodyear übernommen oder neu entdeckt und anschließend weiterentwickelt wurde, konnten die „Krankheiten“ des Kautschuks behandelt, wenn auch nicht vollständig geheilt werden.

Auch zeitgenössische Kautschukhistoriker ziehen aus diesen Fakten bisweilen den Schluss, dass der indianische Stoff , aus dem Wald‘ beträchtliche Probleme aufwies, die eine Karriere in der Alten Welt verhindert hätten, wenn nicht die europäischen oder amerikanischen Erfinder das Material durch Vulkanisation geheilt hätten. So schreibt etwa der Historiker Michael Edward Stanfield in einem Buch, das die Ausbeutung der Indianer in den Mittelpunkt stellt, dass der Kautschuk, den die Indianer erfunden hatten, Schattenseiten hatte, die erst durch anglo-amerikanische Erfinder überwunden wurden:

„But rubber presented some problems, too. Its texture and elasticity changed with temperature: it became hard and brittle in the cold, and soft and tacky in the heat. During the first half of the nineteenth century, Europeans and Americans intensified their effort to stabilize or ,improve" rubber." ${ }^{\text {"68 }}$

Ähnliche Zitate finden sich vielerorts in der Kautschukliteratur. Und doch ist die Aussage falsch. Es ist zwar zutreffend, dass europäische und amerikanische Erfinder ein Verfahren entwickelten, das die Probleme des Kautschuks auf neue Weise löste und ermöglichte, sowohl aus frischem Latex, also aus dem von Hevea brasiliensis und anderen Pflanzen gelieferten Saft, wie auch aus getrocknetem Latex haltbare Gummiprodukte herzustellen.

Falsch ist aber die Ansicht, dass die Europäer und Nordamerikaner die ersten und einzigen waren, denen dies gelang. Diese verbreitete Periodisierung

65 Dargestellt z.B. bei Austin Coates, The Commerce in Rubber. The First 250 Years. Commissioned by the Singapore International Chamber of Commerce Rubber Association, Oxford u. New York 1987, S. 22-28 u. 37-42.

66 Ebd., S. 38f.

67 R.W. Lunn, Vulcanisation, in: Philipp Schidrowitz u. Thomas R. Dawson (Hg.), History of the Rubber Industry, Cambridge 1952, S. 23-39, hier S. 23.

68 Michael Edward Stanfield, Red Rubber, Bleeding Trees. Violence, Slavery, and Empire in Northwest Amazonia, 1850-1933, Albuquerque 1998, S. 20. 
der Kautschukgeschichte bedarf der Korrektur. Übersehen wird hier, dass die süd- und mittelamerikanischen Indianer über ein autonomes Kautschukwissen verfügten. Kern dieses Wissens ist die Kenntnis eines Transformationsverfahrens, das den Kautschuk trocknet und stabilisiert und ihn robust gegen Hitze, Sonnenlicht und Keimbefall macht. Dabei handelt es sich um eine besondere Form der Räucherung, auf die später noch genauer eingegangen wird. Die Indianer verfügten damit über ein funktionales Äquivalent zur Vulkanisation; die indigenen Produkte hatten daher die oben geschilderten Probleme nicht. Unter einem funktionalen Äquivalent verstehe ich dabei einen technischen Prozess, der die zu einer historischen Zeit wahrgenommenen Probleme eines Materials ebenso befriedigend oder besser löst wie eine alternative zeitgenössische Technik. Damit ist nicht gesagt, dass moderne Vulkanisation schlechthin äquivalent mit indigenen Prozessen ist, wohl aber, dass die indigene Prozesstechnik die wichtigsten im frühen 19. Jahrhundert wahrgenommenen Probleme von Kautschukprodukten ebenso gut löste wie die Vulkanisation. Diese Behauptung kann anhand von historischen Aussagen und Fakten belegt werden.

So schreibt William Geer in seiner Kautschukhistoriografie zunächst:

„So completely (sic!) had rubber goods failed the American people because of warm summers and cold winters, that down to about 1840 they were filled with dislike for anything that related to ,gum elastic.' People had become disgusted and rightly so with goods that hardened like a rock in winter and melted in summer."69

Geer stellt aber zugleich klar: „the only reliable articles were Indian-made shoes from the Amazon. “70

Es gab also nach dieser Aussage vor der industriellen Nutzung der Vulkanisation sowohl zerfließende, unbrauchbare Kautschukwaren wie auch gut verwendbare, und diese letzteren wurden von den Indianern im Amazonasgebiet gefertigt. Dass die indianischen Produkte nicht mit Mängeln wie dem Schmelzen bei sommerlicher Hitze behaftet waren, wundert nicht, es wäre im Gegenteil unverständlich, wenn die Indianer jahrhundertelang Kautschukprodukte hergestellt und genutzt hätten, welche derart gravierende Probleme aufgewiesen hätten. Gerade wärmebeständig und beständig gegen Fäulnis mussten die Schuhe und andere indigene Kautschukprodukte ja sein, sonst hätten sie in den Tropen kaum nützlich sein können.

69 William D. Geer, The Reign of Rubber, New York 1922, S. $7 f$.

70 Ebd., S. 9; siehe auch Salo Vincour Coslovsky, The Rise and Decline of the Amazonian Rubber Shoe Industry. A Tale of Technology, International Trade and Industrialization in the early 19th Century, Working Paper 39, MIT 2006, S. 10 und passim. Online unter: http://web. mit.edu/sts/pubs/pdfs/MIT_STS_WorkingPaper_39_Coslovsky.pdf [Stand: 27.10.2013]. 


\section{Handel mit indigenen Kautschukprodukten im 19. Jahrhundert}

Auch andere sachkundige Autoren bestätigten die Qualität der indianischen Produkte. Goodyear selbst würdigt die indigenen Produkte in seiner eigenen Werbeschrift eingehend und glaubt nicht, dass seine eigenen Errungenschaften sie überall ersetzen können. Trotz ihrer Unvollkommenheiten seien indigene Kautschukprodukte, auch ganz ohne all die Verbesserungen, die seine Erfindungen ermöglichten, ,almost indispensable to man. "“1 "The bottles, shoes, and toys made of it [i.e. native gum, J.S.] by the Indians of Para, were exceedingly useful.“72 Ähnlich lautet die Aussage von Henry Wickham, der vor allem durch seinen heimlichen Export von 7.000 Kautschuksamen, der das brasilianische Monopol brach, bekannt ist. Wickham war ein erfahrener Kautschukpflanzer und kannte sich mit der Verarbeitung gut aus. Sowohl die nach den neuen Verfahren vulkanisierten Kautschukprodukte kannte er, wie auch die indianischen. Sein Urteil ist folgendes:

„That the antiseptic smoke-cure is, and will prove to be, the true method for insuring the production of a rubber retaining the characteristic quality of strenght and durability under wear and tear and atmospheric variation, together with the important point of being homogeneous and of even quality throughout, I am convinced. [...] the forest Indian tribes of tropical America [...] have from a time long anterior to the incursion of the Spaniard, been in the habit of making rubber goods for their own use - [...] of a quality for strength and durability excelling any European factory-made. In fact, European factory-made rubber perishes so rapidly in these equatorial forests as to become quite soon useless. "“73

Die indigenen Produkte konnte man in Belém (früherer Name: Pará) bis zur Mitte des 19. Jahrhunderts noch überall kaufen, insbesondere die Gummischuhe. ${ }^{74}$ Überall um Belém herum wurde damals Latex gesammelt und wurden Kautschukwaren produziert, um den Markt zu versorgen. ${ }^{75}$ Gerade die Existenz eines internationalen Handels mit den indigenen Produkten ${ }^{76}$ unterstützt Wickhams Aussagen zur Qualität des Kautschuks, denn dieser Handel wäre wohl nie aufgekommen, wenn die Produkte nicht solide und stabil gewesen wären. Ernesto Cruz widmet dem Handel mit indigenen Kautschukprodukten in seiner História da Associação Comercial do Pará ein Kapitel und belegt anhand von Ausfuhrstatistiken die Produktion und den

71 Goodyear (wie Anm. 6), S. 23.

72 Ebd.

73 Henry Wickham, On the Plantation, Cultivation, and Curing of Para Indian Rubber (Hevea Brasiliensis) with an Account of its Introduction from the West to the Eastern Tropics, London 1908, S. 30, vgl. auch S. 57.

74 Daniel P. Kidder u. James C. Fletcher, Brazil and the Brazilians, Portrayed in Historical and Descriptive Sketches, London 1857, S. 552.

75 Ebd.

76 Philipp Schidrowitz u. Thomas R. Dawson (Hg.), History of the Rubber Industry, Cambridge 1952, S. XIII. 
Export von Gummischuhen aus Pará von der zweiten Hälfte des 18. bis zur Mitte des 19. Jahrhunderts. ${ }^{77}$ Zunächst wurde nach Portugal geliefert, wobei auch Großlieferungen von gummibeschichteten Tornistern für den Gebrauch der portugiesischen Armee belegt ist, so wurden etwa 17972.250 solcher Tornister nach Lissabon ausgeführt. ${ }^{78}$ Von Portugal aus verbreitete sich die Kenntnis der indigenen Gummiprodukte weiter in Europa und nach Nordamerika, die Statistiken zeigen, dass die Ausfuhr in den Jahren der industriellen Einführung der Vulkanisation ihren Höhepunkt erreichte. So wurden von Pará aus im Jahr 1850 138.873 Paar Gummischuhe ausgeführt. ${ }^{79}$ In den Jahren 1854 und 1855 brach der Handel dann ein, und kam in den folgenden Jahren vollständig zum Erliegen. ${ }^{80}$

Der Einbruch lässt sich erklären durch die industrielle Nutzung der Vulkanisation, durch die in Amerika und Europa aus dem Rohmaterial Kautschuk preiswertere und modischere Produkte vor Ort hergestellt werden konnten, die hinsichtlich ihrer Beständigkeit mit den indianischen Produkten dank der neuentwickelten Technologie konkurrieren konnten.

Aber wie war es den Indianern möglich, ihre Kautschukprodukte ohne Schwefel, den es in elementarer Form in Amazonien nur an ganz wenigen Stellen gibt, beständig gegen Hitze, Kälte und Fäulnis zu machen? 1999 zeigten Hosler et al. in einer viel beachteten Untersuchung, dass der von Castilla elastica geerntete Latex mit Saft von Ipomoea alba, einer Sorte der bei uns als Zierpflanze beliebten Prunkwinde, versetzt wurde, wodurch eine Koagulation des Latex herbeigeführt und die elastischen Eigenschaften des entstehenden Kautschuks verbessert werden. ${ }^{81}$ Der Kautschuk der Azteken und der Maya sowie weiterer mittelamerikanischer Völker wurde nicht, wie in Südamerika, aus dem Saft von Hevea brasiliensis, sondern aus dem Milchsaft von Castilla elastica gewonnen.

Ich möchte im Folgenden nachweisen, dass der mesoamerikanischen Verarbeitungstechnik, die von Hosler et al. zurecht ins Licht gerückt wurde, eine amazonische an die Seite zu stellen ist, die weitaus stärker genutzt wurde. Es handelt sich, wie bereits erwähnt, um ein Verfahren der Räucherung.

77 Ernesto Cruz, História da Associacao Comercial do Pará. Centenário de sua fundacao 1864-1964, Belém do Pará 1964, S. 81-83.

78 Ebd., S. 83.

79 Ebd., S. 82f.; vgl. auch John Esaias Warren, Para. Or Scenes and Adventures on the Banks of the Amazon, New York 1851, S. 15: „It has been estimated that at least two hundred and fifty thousand pairs of shoes are annually exported from the province, and the number is constantly on the increase." Coslovsky (wie Anm. 70), S. 14-16 zitiert aus anderen Quellen noch weitaus höhere Zahlen, ebenso Thomas Hancock, Personal Narrative of the Origin and Progress of the Caoutchouc or India-Rubber Manufacture in England, London 1857. Zitiert nach A Centennial Volume of the Writings of Charles Goodyear and Thomas Hancock, Boston 1939, S. 158-165.

80 Cruz (wie Anm. 77), S. 83.

81 Vgl. Hosler et al. (wie Anm. 11). 
Geräucherte indianische Kautschukprodukte waren sowohl hochelastisch wie auch beständig. Sie hatten jene „Krankheiten“ nicht, welche die in Europa und Nordamerika hergestellten Kautschukprodukte aufwiesen.

Der Latexsaft wurde dabei über einem Glimmfeuer aus jungen Zweigen und Urucarí-Nüssen (von der Palme Attalea excelsa) oder Inajá-Nüssen (von der Palme Attalea maripa, auch andere Palmnüsse scheinen verwendet worden zu sein $)^{82}$ zugleich eingetrocknet und chemisch transformiert.

Eine Beschreibung, die 1851 entstand, schildert den indigenen Prozess im Amazonas-Delta auf der Flussinsel Gurupá:

„A fire is made on the ground of the seed of nuts of a palm-tree, of which there are two kinds: one called urucarí, the size of a pigeon's egg, though longer; and the other inajá, which is smaller. An earthen pot, with the bottom knocked out, is placed, moth down, over the fire, and a strong pungent smoke from the burning seeds comes up through the aperture in the bottom of the inverted pot. The maker of the rubber now takes his last, if he is making shoes, or his mould, which is fastened to the end of a stick; pours the milk over it with a cup, and passes it slowly several times through the smoke until it is dry. He then pours on the other coats until he has the required thickness; smoking each coating until it is dry. Moulds are made ether of clay or wood; if of wood, it is smeared with clay, to prevent the adhesion of the milk. When the rubber has the required thickness, the moulds are either cut out or washed out. ${ }^{\text {“83 }}$

Die Technik hat sich auch später kaum geändert. ${ }^{84}$ Wie die Verzierungen angebracht wurden, beschreibt ein anderer Reisender:

„You will, moreover, notice a number of Indian girls (some very pretty) engaged in making various impressions, such as flowers \&c., upon the soft surface of the rubber, by means of their thumb nails, which are especially pared and cultivated for this purpose. After this final operation, the shoes are placed in the sun to harden, and large numbers of them may be seen laid out on mats in exposed situations. ${ }^{685}$

Henry Pearson, der Herausgeber der India Rubber World berichtet, dass der stark riechende Rauch der Nüsse im Wald die Lager der Kautschuksammler schon von Weitem anzeige. ${ }^{86}$ Das Verfahren war also recht geruchsintensiv und zweifellos gesundheitsschädlich. Aber es erfüllte seinen stofflichen Zweck, es „heilte“ den Kautschuk. Es machte ihn zu dem robusten, haltbaren Produkt, das Wickham oben preist.

82 Vgl. Wickham (wie Anm. 73), S. 31.

83 Lewis Herndon, Exploration of the Valley of the Amazon. Made under Direction of the Navy Department, Washington 1853.

84 Vgl. Harald Sioli, Gelebtes, geliebtes Amazonien. Forschungsreisen im brasilianischen Regenwald zwischen 1940-1962, hg. von Gerd Kohlhepp, München 2007, S. 91-93.

85 Warren (wie Anm. 79), S. 17.

86 Henry C. Pearson, The Rubber Country of the Amazon, New York 1911, S. 68. 
Die Räucherung ist der Kern der indigenen Produktionstechnik; dieser Technik entspricht ein besonderes Know-how und auch ein Know-that. Denn es ist klar, dass das indigene Verfahren wissensbasiert ist, es ist schließlich keine Instinkthandlung, sondern wurde von einer Generation auf die andere durch Unterweisung (und nicht durch Gene!) weitergegeben. Das Räuchern findet auf ganz besondere Weise und unter Nutzung spezieller Materialien statt, nur diese hochgradige Selektivität macht es erfolgreich.

\section{Chemische Vermutungen zur Wirksamkeit des indianischen Verfahrens}

Die Räucherung von Kautschukprodukten überträgt vermutlich die beim Räuchern von Fisch, Fleisch oder von Häuten zur Garung und Konservierung eingesetzte Technik auf einen neuen Bereich. Auch dort konserviert das Räuchern und bewirkt zugleich eine Stofftransformation. ${ }^{87}$ Bei der Übertragung des Prinzips entstand aber etwas Neues, denn eine Flüssigkeit zu räuchern ist etwas anderes, als ein fertiges Ding in den Rauch zu halten. Durch das Auftragen immer neuer dünner Flüssigkeitsfilme wurde sichergestellt, dass der Latex durch und durch und nicht nur oberflächlich in Kontakt mit dem speziellen Rauch kam. Man ließ nicht den geformten Latexsaft trocknen und räucherte ihn dann, sondern man räucherte dünne Flüssigkeitsfilme, um die gewünschte Wirkung zu erzielen.

Weshalb war dieses Verfahren so wirksam, dass indianische Kautschukprodukte, wie gezeigt, in der ersten Hälfte des 19. Jahrhunderts international begehrt waren? Wir wissen nicht, wie die Indianer diese Wirksamkeit erklären. Aber wie können wir uns dies erklären? Dabei bringen wir unser eigenes, chemisches Wissen ein. Insofern hierdurch unser Verständnis der Prozesse vertieft werden kann, scheint es sinnvoll, hierzu einige Vermutungen zu wagen.

Es ist zu vermuten, dass durch die Räucherung eine chemische Transformation stattfand, die sich nicht nur auf die Oberfläche des Produktes beschränkte, sondern den Kautschuk durch und durch veränderte. Diese chemische Transformation machte die indianischen Gummiprodukte beständig gegen Hitze, Sonnenlicht und Fäulnis.

Chemisch betrachtet, dürfte die beschriebene Behandlung mindestens folgende sechs Effekte haben: 1) Sie führt zur Koagulation der Kautschuktropfen, sorgt also für eine Phasentrennung, 2) sie bewirkt, dass das Wasser und die weiteren Inhaltsstoffe entfernt werden. Schon das Aufstreichen auf den Ton bewirkt eine Trennung, weil der Ton das Wasser einsaugt; zurück bleibt ein Kautschukfilm. Die durch die Hitze und den Rauch herbeigeführte Koagulation der Kautschukkügelchen, die gern aneinanderkleben, verstärkt dann die Phasentrennung. 3) Die Behandlung konserviert den Kautschuk gegen Keime, 4) sie schützt ihn vor dem Angriff von Luftsauerstoff und 5)

87 Lázlo Tóth u. Reiner Wittkowski, Das Räuchern - aus der Sicht der Chemie, in: Chemie in unserer Zeit 19, 1985, S. 48-58, hier S. 57f. 
UV-Licht und bewirkt 6) eine chemische Umwandlung, die zu einer Steigerung der Elastizität führt, indem die Polyisoprenketten des Latex vernetzt werden.

Rauch ist chemisch gesehen eine ungeheuer komplexe Angelegenheit, vor allem dann, wenn er, wie hier, aus unvollständigen Verbrennungsprozessen ganz bestimmter Hölzer und Nüsse stammt. Das Abdecken des Feuers mit einer oben offenen Schale hat den Effekt, die Sauerstoffzufuhr zu mindern, soweit, dass das Feuer nur noch „kokelt“", aber nicht mehr brennt. Die üblichen Verbrennungsprodukte Wasserdampf und $\mathrm{CO}_{2}$ treten bei unvollständigen Verbrennungen zurück, an ihre Stelle tritt eine kaum übersehbare Fülle komplexer organischer Verbindungen: „Die während der Verglimmung - der sogenannten Pyrolyse - des Holzes entstehenden und sehr zahlreich vorhandenen Verbindungen stellen [...] einen Querschnitt durch die gesamte organische Chemie dar. ${ }^{688}$ Beim Glimmen herrschen Temperaturen von 200 bis 600 Grad Celsius. ${ }^{89}$ Rauch ist eine chemische Allzweckwaffe, ein omnipotentes Reagens, eine materia universalissima gewissermaßen. Dass zu seinen Inhaltsstoffen auch Substanzen gehören, die mit den Polyisoprenketten in der Latexmilch reagieren, ist sicher.

Der besondere, von den Indianern genutzte Rauch wurde vor über 100 Jahren analysiert, ${ }^{90}$ allerdings mit ganz unzulänglichen Methoden (so wurde nur das Destillat und der Rückstand der Verbrennung betrachtet, die Partikel im Rauch und die Gasphase blieben unbeachtet). Immerhin zeigte sich, dass er dem Rauch des bei uns zum Räuchern verwandten Buchenholz ähnelt. Daher kann mangels moderner Untersuchungen zum speziellen Rauch der Nüsse hilfsweise mit Untersuchungen mit Buchenräucherrauch gearbeitet werden. Zwar ist sicher, dass der Rauch der ölreichen Nüsse besondere Bestandteile aufweist, ihm wird von den Indianern besondere Stärke zugeschrieben..$^{91}$ Dennoch galt er nicht als unersetzlich. Auch in Amazonien wird, wo die Nüsse nicht vorhanden sind, mit Hartholzrauch gearbeitet. Mit modernen Methoden wurden in Buchenholzrauch 412 organische Verbindungen nachgewiesen. ${ }^{92}$ Die Zahl der insgesamt vorkommenden Verbindungen wurde auf 10.000 geschätzt. ${ }^{93}$ Neben Ruß enthält der Rauch insbesondere Formaldehyd und andere Aldehyde, Ketone, Ameisensäure, Essigsäure sowie höhere Säuren,

88 Ebd., S. 48.

89 Lázlo Tóth, Chemie der Räucherung. Wissenschaftliche Arbeitspapiere der Senatskommission zur Prüfung von Lebensmittelzusatz- und -inhaltsstoffen, Weinheim 1983, S. 79.

90 Fritz Frank u. Johann Gnädiger, Untersuchungen über die Urukuri-Früchte, in: GummiZeitung 24, 1910, S. 1328-1329.

91 Pearson (wie Anm. 86), S. 70.

92 Tóth/Wittkowski (wie Anm. 87), S. 50; vgl. für eine Zusammenstellung neuerer Untersuchungen Iinuma et al, Source Characterization of Biomass Burning Particles. The Combustion of Selected European Conifers, African Hardwood, Savanna Grass and German and Indonesian Peat, in: Journal of Geophysical Research 112, 2007, S. 2.

93 Tóth (wie Anm. 89), S. 38. 
Methanol und Phenole in beträchtlicher Menge und in vielen Varianten; ${ }^{94}$ hinzu kommen nitrose Gase, also Stickstoffoxide. ${ }^{95}$

Die Säuren im Rauch lassen die Latexmilch koagulieren und wirken antimikrobiell. Unter den weiteren im Rauch vorkommenden Verbindungen sind als aktive Reagenzien besonders wichtig die Gruppe der Phenol-Verbindungen, deren Destillat zusammenfassend als Kreosot (aus dem Griechischen: Fleischretter) bezeichnet wird. Diese sind zum einen stark antimikrobiell, weshalb sie den Kautschuk konservieren. Auch die pH-Absenkung und die Trocknung wirken bereits gegen Mikroorganismen. Unter den Stoffen wirkt besonders die im Rauch enthaltene Salicylsäure, eine Phenolsäure, die ein vielgebrauchtes Konservierungsmittel ist. ${ }^{96}$ Die Phenole schützen den Kautschuk auch gegen Sauerstoff, der ihn brüchig machen würde, sie sind Antioxidantien. ${ }^{97}$ Sie haben aber noch eine dritte wichtige Funktion, denn sie vernetzen die Polyisopren-Ketten miteinander. ${ }^{98}$ Phenol-FormaldehydKondensate werden auch in der modernen Kautschukchemie häufig als Vernetzungsagenzien eingesetzt.

Auch die im Rauch vorhandenen Aerosole, insbesondere der Ruß, haben eine wichtige chemische Funktion. Ruß kommt im Rauch von Schwelfeuern in beträchtlicher Menge vor, aus $100 \mathrm{~g}$ Holz können 1,85 g Ruß entstehen. ${ }^{99}$ Rußpartikel schützen vor UV-Strahlen und sind damit ein Alterungsschutz. Sie fangen UV-Strahlen ab und wandeln sie in Wärme um. So schützen sie das darunterliegende Polymer. Der Einsatz von Ruß als Zusatzstoff von Kautschuk ist heute übliche Praxis; er wird auf Mastizierwalzen in den Gummi eingemischt; dies ist der Grund, weshalb Gummireifen in der Regel schwarz sind.

Zwar war es durch die indigenen Techniken nicht möglich, aus Latex Hartgummi (Ebonit), ein hochgradig vernetztes Produkt, zu fertigen. Dennoch gelang es, mit den indigenen Techniken die wesentlichen Ziele der Bearbeitung zu erreichen: Beständigkeit und gesteigerte Elastizität. Und dies, indem Rohstoffe genutzt wurden, die im Regenwald vorhanden waren. Die indigene Kautschuktechnologie war also eine optimale technische Problemlösung, abgestimmt auf eine bestimmte Produktionsumgebung, da sie mit den

94 Ebd., S. 32.

95 Ebd., S. 51. Zum Mechanismus der Stickoxidbildung bei Temperaturen, wie sie in Glimmfeuern vorkommen vgl. Jürgen Warnatz, Ulrich Maas u. Robert W. Dibble, Verbrennung. Physikalisch-Chemische Grundlagen, Modellierung und Simulation, Experimente, Schadstoffentstehung, 3. Auflage, Berlin u.a. 2001, S. 262-265.

96 Tóth (wie Anm. 89), S. 49, siehe auch S. 90.

97 Ebd., S. 90.

98 Spektroskopische Untersuchungen am ungeronnen und am geronnenen Latex sowie an indianischen Kautschukartikeln konnte ich nicht durchführen, sie wären zur Ergänzung der Untersuchung nützlich, auch wenn sich die Zahl solcher Quervernetzungen erst ab einer gewissen Anzahl mit NMR-Spektroskopie bestimmen lässt, vgl. Hosler et al. (wie Anm. 11), S. 19-89.

99 Tóth (wie Anm. 89), S. 51. 
vorhandenen Mitteln - Latex, Lehm und Feuer - ein Produkt hervorbrachte, das allen Anforderungen standhielt und im 18. und 19. Jahrhundert sogar exportiert wurde.

\section{Und Goodyear? Die Bedeutung der Vulkanisation}

Die in der Literatur vielzitierte Klebrigkeit und Fäulnisanfälligkeit früher amerikanischer oder europäischer Industrieprodukte aus Kautschuk war gerade kein Merkmal indigener Kautschukprodukte. Sie war vielmehr ein Merkmal nur jener Kautschukprodukte, die in Nordamerika oder in England hergestellt wurden. Warum?

Frühzeitig hatte man sich nicht mehr mit den Fertigprodukten aus Brasilien begnügt, sondern versucht, aus der Rohmasse selbst Produkte zu formen. Es war naheliegend, die indigenen Fertigprodukte durch selbstgemachte Erzeugnisse zu ersetzen. Hierbei ging man meist von dem sogenannten Gummispeck, einem nicht geräucherten, sondern getrockneten Latex aus, den man mit Lösungsmitteln (Terpentin) aufquellen ließ. Die Paste strich man zum Beispiel auf Textilien auf. Mit dem Sonnenlicht gelangte dann UV-Licht auf diese Produkte, dieses bewirkte, dass der Kautschuk klebrig wurde. Denn den so verfertigten Waren fehlte der Schutz gegen Sonnenstrahlen und gegen Mikroorganismen, den die ursprünglichen indianischen Produkte aufgrund der im Rauch vorhandenen Stoffe besaßen.

Es waren die in Europa oder Nordamerika aus Gummispeck gefertigten Sekundärprodukte, nicht die eigentlich indianischen Produkte, welche die beschriebenen Qualitätsmängel hatten. Und diese Mängel ${ }^{100}$ rührten in der Regel daher, dass die indianische Oberflächenbehandlung, das Räuchern, welches vernetzt und durch die im Rauch enthaltenen Rußpartikel und Substanzen gegen UV-Licht, Mikroben und Sauerstoff schützt, in Europa oder Nordamerika nicht durchgeführt wurde. Vielmehr ließ man importierten Kautschuk aufquellen, strich ihn dann auf Textilien aus und ließ das Ganze trocknen. Zunächst war das Ergebnis recht passabel. Durch Mikroorganismen oder Sonneneinwirkung verwandelten sich diese Produkte leicht in eine klebrige oder stinkende Masse.

Die Vulkanisation genannte Stofftransformation wird nach dem von Lüdersdorff, Hayward, Goodyear und anderen entwickelten Verfahren heute meist durch gelindes Erhitzen mit Schwefel bewerkstelligt. Dabei wirkt der Schwefel als Vernetzungsagens, das die Polyisoprenketten miteinander verbindet. Oft wird zum Schutz vor Alterung infolge von Lichteinwirkung nach einer späteren Entdeckung von S.C. Mote im Jahre 1904 zusätzlich $\mathrm{Ruß}$ hinzugegeben ${ }^{101}$ oder andere Lichtschutz- und Antioxidationsmittel. So wird die Sauerstoff- und Wärmebeständigkeit erhöht. Dieser Prozess hatte

100 Vgl. die Beschreibung bei Lüdersdorff (wie Anm. 5), S. 19 und passim.

101 Schidrowitz/Dawson (wie Anm. 76), S. 5. 
deshalb so hohe Bedeutung für die europäische Kautschukindustrie, weil er in Kombination mit der zuvor von Hancock entwickelten Mastikationstechnik ermöglichte, mit Kautschuk formend umzugehen und die geformten Gegenstände dann durch Vulkanisation im gewünschten Grade elastisch zu machen. Die indigenen Völker kannten diesen Prozess nicht. Sie brauchten ihn auch nicht, denn sie konnten direkt vom Latex ausgehen. Genau dieser Weg war den europäischen und nordamerikanischen Anwendern aber verschlossen, wie Lüdersdorff in seiner Abhandlung über das „Federharz" klarstellt:

„Man ließ [...] den Pflanzensaft der Siphonia elastica [=Hevea brasiliensis, J.S.] [...] kommen. Natürlich konnte dies nur in festverschlossenen metallenen Gefäßen, oder in Gefäßen von Federharz selbst geschehen. Dadurch aber wurde die Sache wiederum so vertheuert, daß, obwohl mit diesem Safte alle technischen Anforderungen zu erfüllen waren, auch diese Methode nothgedrungen verlassen werden mußte. “102

Der Latex schimmelt ähnlich leicht wie Milch; denn mit seinem Eiweißgehalt und mit seinem Gehalt an Salzen und Wasser ist er ein gutes Nährmedium für Mikroorganismen. ${ }^{103}$ Aus technischen und ökonomischen Gründen waren die europäischen Interessenten darauf angewiesen, vom getrockneten Kautschuk auszugehen. Doch wie sollte man diesen in neue Formen bringen? Hier liegt die eigentliche Leistung der europäischen und amerikanischen Erfinder. ${ }^{104}$ Hancock erfand den Mastikator, der Kautschuk plastisch machte. Nun war der Stoff formbar, war aber klebrig und hatte seine Elastizität verloren. Diese ließ sich mit den Verfahren von Lüdersdorff, Hayward, Goodyear und anderen, der später so genannten Vulkanisation, auf ökonomische und industriell anschlussfähige Weise wieder herstellen. Ein in Europa und den USA billiger, leicht handhabbarer und überall vorhandener Stoff, nämlich der Schwefel, wurde als Problemlösung genutzt, eine genial einfache Lösung. Die Bedeutung der Vulkanisation liegt nicht darin, dass sie aus einem unbrauchbaren indianischen Produkt ein brauchbares machte, sondern darin, dass sie es der amerikanischen und europäischen Industrie ermöglichte, aus getrocknetem Kautschuk oder auch aus recycelten indigenen Produkten selbst haltbare Kautschukprodukte herzustellen und sie damit unabhängiger von den brasilianischen Produzenten machte. Die Abhängigkeit von dem Material sowie vom botanischen indigenen Wissen blieb zunächst noch bestehen. Denn immer noch war man auf die Ortskenntnis und die botanische Kenntnis der Indianer oder Seringuieros (meist Mestizen) angewiesen, da sie in der Lage waren, im Wald die teilweise weit auseinanderstehenden Bäume zu finden und anzuzapfen. Die Abhängigkeit von diesem Wissen wurde erst durch die heimliche Ausfuhr der

102 Lüdersdorff (wie Anm. 5), S. 20.

103 David Blackley, Polymer Latices, 2. Auflage, Bd. 2: Types of Lattices, London u.a. 1997, S. 118.

104 Jean Le Bras, Le Caoutchouc, Paris 1961 (1945), S. 10-14. 
Kautschuksamen durch Henry Wickham im Jahr 1872 gemindert und später durch die industrielle Produktion von synthetischem Kautschuk.

Durch das Auffinden eines Mittels, das die Probleme des Werkstoffs entscheidend minderte, das zudem auch billig und überall verfügbar war, konnte die neue Substanz an die europäische und nordamerikanische industrielle Produktionsroutinen angeschlossen werden und wurde Teil des entstehenden Netzwerks industrieller Massenproduktion.

Dadurch konnten auch neue Qualitäten erreicht werden, die dem indigenen Kautschuk fehlen. Für die Ewigkeit freilich sind auch die modernen vulkanisierten Gummiprodukte nicht gemacht. Denn trotz aller Fortschritte der Kautschuktechnologie ist das Gummiprodukt auch heute noch ein reaktives Polymer, das im Laufe der Zeit, insbesondere, wenn es der Sonne ausgesetzt wird, erst klebrig und dann brüchig wird.

\section{Der Stellenwert indigenen Wissens in der Geschichte des Kautschuks}

Im Rückblick zeigt sich, dass indigenes Wissen für die Geschichte des Kautschuks in dreierlei Hinsicht wichtig war. Zum einen war das Produktwissen erheblich, das Wissen nämlich, wie sich die außergewöhnlichen Eigenschaften in neuartige, nützliche Produkte übersetzen ließen. Viele indigene Produkte wurden von der europäischen und nordamerikanischen Industrie übernommen, wie die Gummischuhe, Gummitiere, Gummiringe und Regenmäntel.

Zweitens war Prozesswissen bedeutsam, nämlich Wissen um Methoden, wie sich die oben erwähnten Produkte aus flüssigem Latex fertigen lassen, und zwar so, dass sie zugleich geschützt waren gegen raschen Verderb durch Fäulnis, Hitze oder Sonneneinstrahlung. Auch dieses Wissen war Voraussetzung für die weitere europäische und nordamerikanische Karriere des Kautschuks, weil ein Stoff, der rasch zerfällt, kaum größeres Interesse erregt hätte. Es waren die stabilen und funktionierenden Produkte, welche für Faszination sorgten. Zwar emanzipierten sich die Europäer und Nordamerikaner rasch vom indigenen Wissen und begannen, ihr eigenes Kautschukwissen aufzubauen. Dennoch blieb das Räuchern als Vorbehandlung zur Qualitätssteigerung in Gebrauch und wurde durch verschiedene Maschinen technisiert.

Der Aufbau eines autonomen europäisch-nordamerikanischen Kautschukwissens hatte starke Rückwirkungen auf die Indianer, die mit der industriellen Umsetzung der Vulkanisation nicht mehr als Produzenten fertiger Waren auftraten. Sie wurden hinsichtlich ihrer sozialen Rolle degradiert, waren nun nur mehr als Rohstoffbeschaffer wichtig und damit in einer weit untergeordneten Rolle tätig. Nicht mehr die durch ihr Stoffwissen garantierte Qualität ihrer Produkte kam in Betracht, sondern nur noch die Quantität des gelieferten Stoffes selbst. Die Grundelemente der indianischen Kautschuktechnologie blieben weiterhin in Gebrauch, insofern der Latex weiterhin Schicht für Schicht über einem Schwelfeuer geräuchert wurde, doch entstanden keine Flaschen, Ringe oder Puppen mehr, sondern nur noch unförmige, möglichst große Ku- 
geln, die damals so genannten „Negerköpfe“ (negro heads). ${ }^{105}$ Keine aus Ton gefertigten Formen wurden eingesetzt, vielmehr wurde der Latex meist auf Paddel gegossen - auf die Form kam es nicht mehr an. Die so entstehenden Kautschukkugeln waren Rohmaterial für westliche Industrien. Auch heute noch werden sie in Amazonien gefertigt, Naturkautschuk ist immer noch für anspruchsvolle Anwendungen unentbehrlich und macht in der Summe etwa ein Drittel der internationalen Kautschukproduktion aus, der größte Teil des Kautschuks wird heute jedoch synthetisch hergestellt.

Dem ersten, von Columbus nach Sevilla mitgebrachten Gummiball folgten bis ins 18. Jahrhundert einzelne weitere Produkte, Ende des 18. und Anfang des 19. Jahrhunderts dann Großlieferungen indigen gefertigter Gummiwaren, ab der Mitte des 19. Jahrhunderts schließlich eine wahre Springflut von kleineren und größeren Bällen, die aber nicht mehr zur unmittelbaren Verwendung, sondern nur mehr als Rohstoff genutzt wurden. Diese wurden nicht mehr nach Sevilla verschifft, sondern zu den großen Industriehäfen Europas und Nordamerikas.

Die dritte Dimension des indigenen Kautschukwissens, das Wissen nämlich, welche Pflanzenarten verwertbaren Latex führen, wo sie zu finden sind und wann sie besonders viel Kautschuk führen, blieb für die Europäer und die Nordamerikaner auch im späteren 19. Jahrhundert noch wichtig, weil in den polyspezifischen Urwäldern Amazoniens auf einem Hektar bis zu 150 Baumarten stehen können und die Kautschukbäume oft weit auseinanderliegend wachsen. ${ }^{106}$ Sie können von Mai bis Oktober geerntet werden, wobei sie Milch vor allem morgens geben. ${ }^{107}$ Auch an einem zentralen Wendepunkt der Kautschukgeschichte war dieses indigene Wissen entscheidend: Henry Wickham, der die Kautschuksamen aus Brasilien ausführte und damit das brasilianische Kautschukmonopol brach, hätte diese umstrittene Tat nämlich ohne das botanische und biogeografische Wissen der Tapüyo-Indianer, die zwischen dem Rio Madeira und dem Rio Tapajós für ihn die rund 7.000 Samen genau nach der geforderten Varietät sammelten und fachgerecht verpackten, wie er selbst schreibt, ${ }^{108}$ nicht vollbringen können.

Durch den Aufbau autonomen Kautschukwissens in Europa und Nordamerika entwickelten sich die Indianer von kompetenten Gummitechnikern zu „Seringueiros“ zurück, zu Kautschukzapfern; und auf dem Höhepunkt des brasilianischen Kautschukbooms, im ausgehenden 19. Jahrhundert, wurden die Indianer vielerorts zwangsverpflichtet, am Putumayo in Nordwestamazonien kam es zur Errichtung eines ungeheuerlich grausamen Systems der

105 Pearson (wie Anm. 86), S. 57.

106 Ebd., S. 61-67; Sioli (wie Anm. 84), S. 91.

107 Heinrich Johann Reimers, Reise-Führer von Europa nach Brasilien, Hamburg 1914, S. $90 f$.

108 Wickham (wie Anm. 73), S. 48-52. 
Zwangsarbeit für die Peruvian Rubber Company. ${ }^{109}$ Statt der durchgebildeten und verzierten Schuhe, Flaschen usw., die zuvor exportiert wurden, kamen aus den Wäldern nun nur noch riesengroße, unförmige Würste oder 50 Kilo schwere Bälle, ${ }^{110}$ die gerade noch gehoben werden konnten, als Rohstoff für die Weiterverarbeitung in Nordamerika und Europa.

Andererseits aber blieb für den eigenen Bedarf das Anfertigen von Kautschukgegenständen weiterhin in Gebrauch. Dabei wurde nun auch das Kautschukwissen der Weißen adaptiert, wie Henry Pearson beschreibt:
„Nearly all of the Indian tribes make rubber ponchos, kit bags, and some very curious toys. In making a rubber bag, they first make a bag of fabric, sometimes of prettily flowered calico, which they stretch over a frame until the surfaces are smooth and taut. Then they take caucho milk, never using Hevea, and stir into it powdered sulphur, the proportion being a tablespoonful of sulphur to each liter of latex. After stirring the liquid thoroughly, they apply it to the cloth with a feather and give it a sun cure. If sulphur is not obtainable they use gunpowder. When the sulphur compound is spread over flowered calico the colors show through and the bags are extremely pretty. The gunpowder mixture, of course is black and not transparent. These bags will outlast a ozen made of vulcanized rubber and are eagerly purchased by engineers and prospectors." ${ }^{\text {"111 }}$

Im Rückblick erscheint die indigene Kautschuktechnologie primitiv. Auch die indigenen Völker Brasiliens scheinen die Technik allenfalls noch in sehr abgelegenen Gegenden zu beherrschen. Neuere Versuche, indigene Völker wieder zur Fertigung von Kautschukwaren anzuregen, werden in Nordwestamazonien unternommen, hier werden als ,nachhaltige Produkte“ indigener Völker zum Beispiel Taschen, Flaschenkühler, Mousepads und anderes vermarktet. Die neuen indigenen Gummiprodukte werden unter anderem unter dem Titel, couro vegetal' vermarktet - pflanzliches Leder - und es ist sehr wohl denkbar, dass die Kombination aus Regenwaldschutz und der Unterstützung indigener Minderheiten diesen Artikeln einen breiten Markt beschert. Auf dem Markt am Rande der Rio+20-Konferenz in Rio de Janeiro erfreuten sich die Waren der Encauchados, wie sich die neuen indigenen Gummiwerker nennen, jedenfalls großer Nachfrage. Die neue Praktik wird von verschiedenen Stiftungen, von der Regierung der Amazonasstaaten Acre, Rondônia und Pará sowie von der brasilianischen Regierung unterstützt, da hier eine Möglichkeit für indigene Gemeinschaften und andere im Regenwald lebende marginalisierte Gemeinschaften geschaffen wird, Einkommen zu erzielen, ohne den Wald,

109 Siehe dazu zuletzt Stanfield (wie Anm. 68). Zu Reflexen jener Zeit in indigenen Mythen siehe Dimitri Karadimas, Animism and Perspectivism: Still anthropomorphism? On the Problem of Perception in the Construction of Amerindian Ontologies, in: Indiana 29, 2012, S. 25-51, hier S. 50 .

110 Reimers (wie Anm. 107), S. $91 \mathrm{f}$.

111 Pearson (wie Anm. 86), S. 129. 
z.B. durch illegalen Holzeinschlag, zu ruinieren. Nach langen Jahren des Niedergangs indigener Kautschuktechnologie sehen manche schon einen neuen „ciclo da borracha na Amazônia“, einen neuen Kautschukboom in Amazonien kommen. ${ }^{12}$ Eine Wiederbelebung indigener Kautschuktechnologie ist zweifellos zu begrüßen und möglicherweise ist die neue ,tecnica social' geeignet, indigenen Kautschuktechnologien wieder Zutritt zum Markt zu verschaffen. Doch die neuen Produktionsformen vitalisieren gerade nicht das alte Wissen, sondern ersetzen es durch angeblich ökologischere und gesündere chemische Präparate ${ }^{113}$ wodurch die Produzenten von den Lieferanten jener Präparate abhängig werden. Immerhin aber zeigen diese Versuche, dass es möglich ist, neue Synthesen von indigenem und westlichem Wissen zu schaffen, und auf deren Grundlage neuartige indigene Kautschukprodukte herzustellen. ${ }^{114}$

Anschrift des Verfassers: Dr. Jens Soentgen, Scientific Director, Wissenschaftszentrum Umwelt, Universität Augsburg, Universitätsstraße 1a, D-86159 Augsburg, Email: soentgen@wzu.uni-augsburg.de.

112 Karine Rodrigues, Couro vegetal abre um novo ciclo da borracha na Amazônia. Online unter: http://www.rts.org.br/noticias/destaque-1/couro-vegetal-abre-um-novo-ciclo-daborracha-na-amazonia [Stand: 30.10.2013].

113 Vgl. die Vorschläge in Assunção Amaral u. Francisco Samonek, Borracha, Amazônica. Arranjos produtivos locais, novas possibilidades e políticas públicas, Paper do Núcleo de Altos Estudos Amazônicos 191, 2006. Online unter: www2.ufpa.br/naea/ [Stand: 27.11.2013].

114 Für inhaltliche Kritik, Hinweise und Unterstützung danke ich Prof. Dr. Klaus Hilbert, PUCRS, Porto Alegre, Dr. Mona Suhrbier, Museum der Weltkulturen, Frankfurt a.M., Dr. Katrin Vogel u. Michael Schweiger, Augsburg und den Gutachtern der Wissenschaftlichen Leitung der Zeitschrift Technikgeschichte. Insbesondere danke ich Dr. Ernst Schwinum, Leverkusen/Bergisch Neukirchen, der mir vor vielen Jahren ermöglichte, im KautschukPrüflabor der Bayer AG einige Wochen mitzuarbeiten, und der mir durch sein außergewöhnliches Fachwissen über Kautschuk, über die Kautschukgeschichte und die Kautschukchemie entscheidend geholfen hat. 